\title{
Synaptic memory requires CaMKII
}

5 Wucheng Tao ${ }^{1,2}$, Joel Lee ${ }^{2 \#}$, Xiumin Chen $^{2 \#}$, Javier Diaz- Alonso ${ }^{2,5}$, Jing Zhou ${ }^{3}$,

$7 \quad{ }^{1}$ Key Laboratory of Brain Aging and Neurodegenerative Diseases, School of

8 Basic Medical Sciences, Fujian Medical University, Fuzhou 350108, Fujian

9 Province, China.

$10{ }^{2}$ Departments of Cellular and Molecular Pharmacology, ${ }^{3}$ Neurology, and

$11{ }^{4}$ Physiology, University of California, San Francisco, CA

12 Present address: Department of Neuroscience, University of California, Irvine,

$13 \mathrm{CA}$

$15 \quad$ "contributed equally

17 *Correspondence should be addressed to:

25 Submission: July 12, 2020 


\section{SUMMARY}

34 Long-term potentiation (LTP) is arguably the most compelling cellular model for

35 learning and memory. While the mechanisms underlying the induction of LTP

36 ("learning") are well understood, the maintenance of LTP ("memory") has

37 remained contentious over the last 20 years. Here, we find that CaMKII

38 contributes to synaptic transmission and is required LTP maintenance. Acute

39 inhibition of CaMKII erases LTP and transient inhibition of CaMKII enhances

40 subsequent LTP. These findings strongly support the role of CaMKII as a

41 molecular storage devise.

\section{INTRODUCTION}

45 The mechanism by which the brain stores information has fascinated 46 neuroscientists for well over a century. Two general ideas have emerged. The 47 first proposes that information is held by ongoing activity in neuron ensembles.

48 This, indeed, underlies short-term working memory (D'Esposito and Postle, 2015;

49 Inagaki et al., 2019; Wang, 2001). However, it is clear that such a mechanism 50 cannot account for long lasting, enduring memories. For instance, it is well

51 established that patients who recover from prolonged periods of isoelectric EEG

52 and brain stem silence following barbiturate overdose have no detectable

53 cognitive deficits, e.g., (Bird and Plum, 1968). The second and generally

54 accepted view, first enunciated by Cajal over a century ago (Cajal, 1911), is the

55 modification of neuronal connections. How these changes might be stored has

56 remained a mystery.

58 Over the past few decades, $\mathrm{Ca}^{2+}$-calmodulin-dependent kinase II (CaMKII) has

59 emerged as a very attractive molecular candidate for information storage (Bayer

60 and Schulman, 2019; Bhattacharyya et al., 2020; Hell, 2014; Kennedy, 2013;

61 Lisman et al., 2002; Lisman et al., 2012; Lisman and Goldring, 1988). This 
62 dodecameric kinase is activated by $\mathrm{Ca}^{2+} / \mathrm{CaM}$ resulting in autophosphorylation 63 and the activity of the kinase remains after the removal of $\mathrm{Ca}^{2+}$ (Miller and 64 Kennedy, 1986), a state referred to as autonomy. Furthermore, evidence 65 suggests that the enzyme can undergo activation-triggered subunit exchange 66 such that subunits that did not experience the original $\mathrm{Ca}^{2+}$ transient are 67 phosphorylated, a mechanism postulated to allow for continued phosphorylation

68 in the face of protein turnover (Bhattacharyya et al., 2020; Bhattacharyya et al., 69 2016; Stratton et al., 2014) Thus, CaMKII has many attractive biochemical 70 features expected for a memory molecule. What is the physiological evidence for 71 such a role?

73 Long-term potentiation (LTP), in which brief high frequency synaptic stimulation 74 results in a lasting increase in synaptic strength, is an attractive cellular model for 75 learning and memory (Choquet, 2018; Collingridge et al., 2004; Huganir and 76 Nicoll, 2013; Malinow and Malenka, 2002; Nicoll, 2017). A role for CaMKII in LTP 77 is compelling. Pharmacologically blocking CaMKII (Malenka et al., 1989; Malinow 78 et al., 1989; Otmakhov et al., 1997) or genetically deleting CaMKII (Giese et al., 79 1998; Incontro et al., 2018; Silva et al., 1992) blocks LTP and constitutively active 80 CaMKII both mimics and occludes LTP (Lledo et al., 1995; Pettit et al., 1994; Pi 81 et al., 2010; Poncer et al., 2002). However, despite the attractive biochemical 82 properties of CaMKII, whether CaMKII is involved in the maintenance of LTP and, 83 by extension, memory storage remains problematic.

85 There are two predictions if CaMKII is responsible for LTP maintenance and 86 synaptic memory (Lisman, 2017; Sanhueza and Lisman, 2013). First, transiently 87 blocking CaMKII after the induction of LTP should cause a long-lasting erasure. 88 Attempts at reversing LTP have failed on numerous occasions (Buard et al., 89 2010; Chen et al., 2001; Malinow et al., 1989; Murakoshi et al., 2017; Otmakhov 90 et al., 1997), but see (Feng, 1995). Second, if CaMKII is involved in synaptic 91 memory, one would expect it to leave a lasting trace at synapses (Lisman, 2017; 92 Sanhueza et al., 2011; Sanhueza and Lisman, 2013). Thus, silencing CaMKII 
93 should reduce synaptic transmission. Interestingly, the CaMKII/NMDAR complex

94 does exist under basal conditions (Leonard et al., 1999). However, the genetic

95 deletion of CaMKII (Achterberg et al., 2014; Giese et al., 1998; Silva et al., 1992),

96 but see (Hinds et al., 1998), or pharmacological inhibition of CaMKII does not

97 affect baseline synaptic responses (Buard et al., 2010; Chen et al., 2001; Feng,

98 1995; Malinow et al., 1989; Murakoshi et al., 2017; Otmakhov et al., 1997; Wang

99 and Kelly, 1996). Finally, using a FRET-based CaMKII sensor, CaMKII activation

100 in spines after LTP induction persists for only $\sim 1$ min. (Lee et al., 2009). Thus,

101 physiological support for a role of CaMKII in maintaining LTP or synaptic

102 transmission is lacking.

103

104 Recent findings have prompted us to reevaluate CaMKII's role in the 105 maintenance of LTP. Deleting CaMKII with CRISPR (Incontro et al., 2018) or 106 expressing a peptide inhibitor of CaMKII (Goold and Nicoll, 2010; Sanhueza et al., 107 2011) cause a substantial reduction in synaptic transmission. Furthermore, 108 studies using a membrane permeable peptide inhibitor of CaMKII (tatCN21 or 109 antCN27) have reported a lasting depression in synaptic transmission (Barcomb 110 et al., 2016; Gouet et al., 2012; Sanhueza et al., 2011; Sanhueza et al., 2007) 111 and evidence for a reduction in LTP maintenance (Sanhueza et al., 2011; 112 Sanhueza et al., 2007).

114 To reevaluate the role of CaMKII in synaptic memory we have used two different 115 rapidly acting and reversible CaMKII inhibitors. As would be expected if LTP 116 contributes to synaptic memory, inhibition of CaMKII depresses synaptic 117 transmission and the properties of this persistent action of CaMKII are 118 remarkably similar to those of LTP. Furthermore, we demonstrate that applying 119 these inhibitors after inducing LTP fully reverses LTP, indicating that CaMKII is 120 required for the persistence of LTP. Thus, our findings strongly support CaMKII 121 as a molecular storage devise. Reasons for why the present results differ from 122 most previous studies are discussed. 


\section{RESULTS}

\section{Blocking CaMKII inhibits synaptic transmission}

127 Previous studies established, both with the expression of inhibitory peptides 128 (Goold and Nicoll, 2010; Sanhueza et al., 2011) or CRISPR deletion of CaMKII 129 (Incontro et al., 2018) that CaMKII contributes 50\% to AMPAR responses. In 130 order to study the basis for this activity as well as the role of CaMKII in LTP 131 maintenance, rapid and reversible block of CaMKII is essential. Since peptide 132 inhibitors have proved most effective, this requires making these peptides 133 membrane permeable either by cell-penetrating peptides (CPPs) (e.g., tat) or by 134 protein lipidation (e.g., myristoylation). Delivery of peptides into cells has 135 profound therapeutic potential and therefore has received a great deal of study

136 (Allen et al., 2018; LeCher et al., 2017; Nelson et al., 2007; Patel et al., 2019).

137 The effectiveness of CPPs is hotly debated, because, although these peptides 138 clearly enter the cell by endocytosis, it is not certain to what degree these 139 peptides actually have access to the cytoplasm. This appears not to be an issue 140 with lipid modification.

142 As an initial assay we bathed acute slices in a peptide inhibitor and measured the 143 AMPAR/NMDAR ratio. We would expect the ratio to be reduced by $\sim 50 \%$, since 144 expressing these peptides had no effect on NMDAR currents. We first tested the 145 effectiveness of tatCN21 (5 $\mu \mathrm{M}$ ) (Barcomb et al., 2016; Buard et al., 2010; 146 Sanhueza et al., 2011) in reducing the AMPAR/NMDAR ratio (Fig. S1). In 147 agreement with previous reports, we failed to see an effect on AMPAR 148 responses. Since higher concentrations of tatCN21 have significant nonspecific 149 effects (Barcomb et al., 2016), we turned to other peptides. Previous work (Goold 150 and Nicoll, 2010) showed that prolonged (1-2 days) incubation with myr-CN27 $151(10 \mu \mathrm{M})$ caused a $\sim 50 \%$ reduction in the AMPAR/NMDAR ratio. We, therefore, 152 repeated this experiment with shorter incubations (1-2 hours) and a lower 153 concentration $(1 \mu \mathrm{M})$ and found that myr-CN27 reduced the ratio by $\sim 50 \%$ (Fig. $154 \mathrm{~S} 1 /$ Figure1-figure suple1). What might account for the lack of effect of tatCN21? 
155 To address this, we repeated the experiment using Myr-CN21 (5 $\mu \mathrm{M})$ and found

156 that it reduced the ratio by $\sim 50 \%$ (Fig. S1/ Figure1-figure suple1), suggesting that

157 lipidation is a more effective mode of delivery than CPPs. We, therefore, selected

158 myr-CN27 for our studies.

160 Application of myr-CN27 to acute slices $(1 \mu \mathrm{M})$ caused a highly reproducible 161 slowly developing depression of synaptic AMPAR mediated EPSCs, which 162 stabilized after approximately $40 \mathrm{~min}$. (Fig. 1A and B). A number of experiments 163 were carried out to ensure that the effect we observe with myr-CN27 is due to the 164 specific inhibition of CaMKII. First, in many experiments we measured the size of 165 the NMDAR EPSC, by depolarizing the cell to $+40 \mathrm{mV}$ before and after the 166 application of the peptide. We found that myr-CN27 had no effect on the NMDAR 167 EPSC (Fig. 1A, red circles, Fig. 1B). Second, it is important to note that the 168 magnitude of the decrease in transmission seen with myr-CN27 is similar to that 169 reported with the genetic deletion of CaMKIla (Incontro et al., 2018) suggesting 170 that myr-CN27 fully silences CaMKII. Finally, we examined the effects of myr171 CN27 in cells lacking CaMKIla (Fig. 1C and D). CaMKIla was deleted using 172 CRISPR (Incontro et al., 2018). We simultaneously recorded from control cells 173 and cells lacking CaMKIla in slice culture. In the absence of CaMKIla (green 174 circle) synaptic responses were $\sim 50 \%$ of control (black circles), as expected. 175 Application of myr-CN27 depressed controls cells to the level recorded in cells 176 lacking CaMKIla (Fig. $1 \mathrm{C}$ and D, black circles), but it had no additional effect on 177 cells lacking CaMKIla (green circles) $(n=6)$. These results demonstrate that the 178 effect of CN27 is fully explained by its selective and complete silencing of 179 CaMKIla.

181 Previous studies have relied on field potential recording and thus we repeated 182 our experiments with this approach (Fig. 1E). We still recorded a depression, but 183 in this case the depression took tens of minutes to develop (black symbols). We 184 interleaved these experiments with whole cell recording and confirmed that the 185 depression is considerably faster (blue symbols). We interpret the difference in 
186 time course to the fact that whole cell recordings are made from superficial cells,

187 while field potential responses are generated by populations of neurons 188 throughout the depth of the slice. Penetration of peptides into the slice is 189 expected to be slow.

191 We next examined the effects of AIP, a peptide inhibitor designed from the 192 autoinhibitory domain. We first expressed AIP in neurons in slice culture and 193 compared AMPAR and NMDAR responses to those in simultaneously recorded 194 neighboring control cells (Fig. 2A). Similar to the effect of expressing CN27 195 (Goold and Nicoll, 2010) or applying myr-CN27 (see above), there was a 196 selective $\sim 50 \%$ reduction in the AMPAR EPSC, but no change in the NMDAR 197 response. Given these positive results, we bathed acute slices in myr-AIP (20 $198 \mu \mathrm{M})$ and measured the AMPAR/NMDAR ratio (Fig. 2B) $(n=12)$. The ratio was 199 significantly reduced. Acute application of myr-AIP in slice culture caused a 200 slowly developing depression in synaptic activity (Fig. 2C, black circles) $(n=6)$ 201 and this effect is due to the selective inhibition of CaMKII, because interleaved 202 recording from cells in which CaMKIla had been deleted (Fig. 2C, green circles), 203 myr-AIP no longer had any effect $(n=5)$. These findings show that myr-CN27 204 and myr-AIP selectively and completely inhibit CaMKII, both in acute slices and 205 in slice culture. It is interesting that, while CaMKII is expressed presynaptically 206 and reported to effect transmitter release (Benfenati et al., 1992; Hinds et al., 207 2003), there was no presynaptic effect of the peptide inhibitors.

209 The time course of inhibition for both myr-CN27 and myr-AIP is slow, requiring 21010 's of minutes. Is this due to the slow access of these peptides to the cell 211 interior, or is it due to the slow reversal of the action of CaMKII? To address this, 212 we turned to a novel light inducible inhibitor of CaMKII, in which the inhibitory 213 peptide AIP2 (Ishida et al., 1998) is linked to the LOV-Ja helix domain of 214 Phototropin (referred to as paAIP2) (Murakoshi et al., 2017). Blue light rapidly 215 exposes the AIP2 peptide, which then returns to its closed inactive conformation 216 in approximately $40 \mathrm{~s}$ following light exposure. We expressed paAIP2 in 
217 individual neurons with biolistics, which, unlike the myristoylated peptides, has 218 the advantage of limiting the manipulation to individual postsynaptic cells. The 219 synaptic properties of neurons expressing paAIP2 were not different from the 220 properties of neighboring control cells (Fig. S2A and B/ Figure3-figure suple1.2A 221 and $B)(n=16)$. Exposure of these cells to blue light resulted in a slowly 222 developing inhibition of AMPAR responses (Fig. 3A) $(n=12)$, similar to the 223 kinetics of myr-CN27 and myr-AIP. The blue light had no effect on 224 simultaneously recorded control cells (Fig. 3A, black circles) or in cells co225 expressing paAIP2 with CRISPR to delete CaMKIla (Fig. S3/ Figure3-figure 226 suple2) $(n=5)$, establishing the specificity of paAIP2. These results with paAIP2 227 suggest that the slow time course is not due to slow access, but rather due to the 228 slow reversal of the action of CaMKII.

\section{The origin of the constitutive action of CaMKII}

232 The persistent CaMKII activity has two possible origins. First, it could represent 233 ongoing constitutive $\mathrm{Ca}^{2+}$ activation of CaMKII from various sources. It seems 234 unlikely that resting $\mathrm{Ca}^{2+}$ levels (Maravall et al., 2000) stimulate CaMKII, since 235 the sensitivity of CaMKII to $\mathrm{Ca}^{2+}$ requires considerably higher levels of $\mathrm{Ca}^{2+}$ 236 (Schulman, 1984). To directly test whether constitutive CaMKII activity is $\mathrm{Ca}^{2+}$ 237 dependent, we loaded cells with the $\mathrm{Ca}^{2+}$ chelator BAPTA (15 mM). However, 238 myr-CN27 retained its depressive effect (Fig. S4A/Figure4-figure suple1.A). This 239 finding also rules out spontaneous background NMDAR activity as a source for 240 the constitutive CaMKII activity. In addition, acute application of the NMDAR 241 antagonist APV, has little effect on AMPAR EPSCs (Incontro et al., 2018).

243 The constitutive activity might reflect an LTP process induced by slice 244 preparation, which is known to depolarize cells and release glutamate. To 245 address this possibility, we pretreated animals with a high dose of MK-801, which

246 is known to block in vivo NMDAR responses (Davies et al., 1988). To verify that 247 NMDARs were blocked by this procedure, we recorded NMDAR EPSCs from 
248 slices from these animals and found that the NMDAR EPSC was blocked (Fig. 249 S4B/Figure4-figure suple1.B). Having confirmed the absence of NMDAR 250 responses the slices were perfused with MK-801. Under such conditions myr251 CN27 still exerted its inhibitory effect (Fig. S4C/ Figure4-figure suple1.C).

253 Second, if CaMKII is involved in synaptic memory, one would expect that LTP 254 acquired when the animal was alive, would contribute to synaptic transmission, 255 as previously proposed (Lisman, 2017; Sanhueza and Lisman, 2013). If this is 256 the case, there are a number of predictions. One would expect that the transient 257 inhibition of CaMKII should result in long lasting inhibition, akin to the resetting of 258 a molecular switch. In a number of cells we monitored the depression over time 259 following the removal of myr-CN27 and found no recovery (Fig. 4A). One might 260 argue that myr-CN27 fails to washout of the cells. To circumvent this possibility, 261 we repeated these experiments with paAIP2 whose action rapidly reverses upon 262 terminating the blue light ( $40 \mathrm{~s})$. Again, we failed to observe any recovery (Fig. 263 4B). We confirmed that this was not due to the continued action of the inhibitor, 264 by demonstrating that LTP could be induced following light termination (see 265 below, Fig. 5).

267 If the constitutive activity resulted from prior NMDAR-dependent LTP, one would 268 expect that this inhibitory action should be absent in cells in which NMDARs have 269 been deleted embryonically. We carried out in utero electroporation to express 270 cre recombinase in neurons in GluN1 floxed mice. Transfected cells were 271 recorded at P16-20 in acute slices. We first carried out paired recordings in the 272 AMPAR inhibitor CNQX to ensure that cre expressing neurons lacked NMDAR 273 EPSCs (Fig. 4C, left panel). Application of myr-AIP had no effect in these cells 274 (Fig. 4C, middle and right panels, green circles and traces), whereas it had its 275 normal inhibitory effect in interleaved control cells (Fig. 4C, black circles and 276 traces), further supporting the conclusion that the persistent CaMKII activity 277 represents prior LTP. A caveat to these experiments is that the synaptic action of 278 CaMKII requires its binding to the GluN2B C-terminus of the NMDAR. Thus, the 
279 loss of GluN2B in the GluN1 lacking neurons could equally explain the loss of 280 effect of myr-CN27. To address this concern we repeated these experiments, 281 replacing GluN1 with a pore dead GluN1 mutant (N812W) (Amin et al., 2017). 282 While this mutant assembles normally with GluN2 subunits and traffics normally 283 to the membrane, it generates little current (Amin et al., 2017). We carried out in 284 utero electroporation in GluN1 floxed mice to transfect Cre and GluN1(N812W). 285 Acute slices were made at P14-18. The NMDAR EPSC in transfected neurons 286 was severely reduced, when compared to neighboring control cells recorded in 287 CNQX (Fig. 4D, left panel). The depressant effect of myr-CN27 recorded in these 288 transfected cells was absent (Fig. 4D, middle and right panels). These results 289 suggest that in behaving animals, cells that lack NMDAR function, but with 290 GluN2B intact, $\mathrm{Ca}^{2+}$ signaling independent of the NMDAR appears incapable of 291 activating CaMKII, at least in regard to its action on synaptic function.

293 Since LTP is saturable, one might expect that when constitutive activity is 294 transiently silenced, the magnitude of LTP should be larger than that in control 295 conditions. To test this prediction, we transfected paAIP2 in utero and prepared 296 acute slices at days P15-25. The slices were exposed to blue light for 40 minutes 297 and then simultaneous recordings were made from a transfected cell and a 298 control cell (Fig. 5A). In a typical experiment (Fig. 5B), as well as in the summary 299 of all experiments (Fig. 5D), the size of baseline EPSCs in paAIP2 expressing 300 cells following blue light exposure was, as expected, approximately half of that of 301 the control neighboring cells. LTP was then induced in both cells. To compare 302 the magnitude of LTP in the two cells we normalized the baseline EPSCs to $303100 \%$. As is clear, both in the single experiment (Fig. 5C) and in the summary 304 (Fig. 5E), the magnitude of LTP in cells expressing paAIP2 is approximately 305 twice that of control cells. This interaction between the constitutive potentiation 306 and LTP is consistent with their sharing the same underlying mechanism. Taken 307 together these results strongly support the conclusion that while the animal is 308 alive, LTP is acquired and leaves a synaptic memory trace. 
311 The experiments presented thus far are all consistent with the notion that 312 synapses acquire LTP that contributes to synaptic transmission. If this is correct, 313 then inhibiting CaMKII after LTP induction must reverse the potentiation. Yet, as 314 discussed in the introduction, this experiment has failed on numerous occasions 315 (Buard et al., 2010; Chen et al., 2001; Malinow et al., 1989; Murakoshi et al., 316 2017; Otmakhov et al., 1997), but see (Feng, 1995). Thus, it was mandatory that 317 we revisit this crucial experiment. We therefore carried out a "two pathway 318 experiment", in which the responses from two independent pathways were 319 recorded from a single cell (Fig. 6A). Following the recording of baseline 320 responses, LTP was induced in one of the pathways (Fig. 6B, red circles) by 321 pairing depolarization of the cell to $0 \mathrm{mV}$ with continued synaptic stimulation, 322 while in the other control pathway, stimulation was stopped during the 323 depolarization (Fig. 6B, black circles). Once LTP had been established, myr324 CN27 was applied and the effect on both pathways recorded. Myr-CN27 caused 325 a complete reversal of established LTP and as expected, a $\sim 50 \%$ reduction in the 326 control pathway (Fig. 6B). A summary of all the experiments (Fig. 6B, $n=11$ ) 327 demonstrates that in the presence of myr-CN27 the LTP pathway and control 328 pathway converge to approximately $50 \%$ of the control. This experiment 329 establishes the essential role of CaMKII in maintaining LTP. A previous field 330 potential study using tatCN21 $(5 \mu \mathrm{M})$ failed to observe a reversal of LTP (Buard 331 et al., 2010). We, therefore, repeated our two pathway experiment using field 332 potential recording. Again, we observed that myr-CN27 $(1 \mu \mathrm{M})$ caused a slow 333 reduction the EPSPs in the control pathway and a reversal of LTP (Fig.S5/ 334 Figure6-figure suple1). Give that tatCN21 (5 $\mu \mathrm{M})$ had no effect on synaptic 335 transmission (Buard et al., 2010) (Fig. S1/ Figure1-figure suple1), it is not 336 surprising that it failed to reverse LTP in the previous study (Buard et al., 2010).

\section{DISCUSSION}


341 The role of CaMKII in the maintenance of LTP and information storage has been

342 one of the most vexing issues in the field of synaptic plasticity. On the one hand,

343 the biochemical properties of CaMKII have for decades made this molecule an

344 extremely attractive candidate for molecular storage (Bhattacharyya et al., 2020;

345 Coultrap and Bayer, 2012; Hell, 2014; Lisman et al., 2002; Lisman et al., 2012).

346 On the other hand, numerous physiological experiments over the years have

347 failed to support the role of CaMKII in the maintenance of LTP and by extension

348 memory. To reevaluate the physiological role of CaMKII in synaptic memory, we

349 have used two classes of CaMKII inhibitory peptides. With these inhibitors we

350 find that CaMKII is required for the maintenance of LTP and provide evidence

351 that LTP acquired while the animal was alive leaves a lasting synaptic memory 352 trace.

353

354 The first class of inhibitory peptide we used is derived from an endogenous

355 CaMKII inhibitory protein referred to as CaMKIINtide (CN27) (Chang et al., 1998;

356 Goold and Nicoll, 2010; Pellicena and Schulman, 2014; Vest et al., 2007). The

357 second class of inhibitory peptide is derived from the autoinhibitory domain of

358 CaMKII (Bayer et al., 2001; Leonard et al., 1999). Although these inhibitory

359 peptides were thought to bind to separate sites ( $S$ and $T$ sites), recent structural

360 studies of the binding of GluN2B and other interacting peptides to CaMKII

361 indicate that these peptides use similar interactions to bind across the substrate

362 binding pocket of the CaMKII active site (Özden et al., 2020). Thus, at present it

363 is not possible to use these two classes of inhibitors to distinguish between the

364 disruption of GluN2B binding and the blockade of kinase activity. These peptides

365 included a myristoylated version of CN27 (myr-CN27), AIP (myr-AIP) and a

366 recently developed photoactivatable peptide inhibitor (paAIP2) (Murakoshi et al.,

367 2017). Both classes of peptides block kinase activity with high affinity (Chang et

368 al., 2001; Ishida et al., 1998), but also interfere with the binding of CaMKII to

369 GluN2B (Sanhueza et al., 2011; Vest et al., 2007). 
371 Our experiments clearly establish that inhibitory peptides fully reverse LTP. How 372 long is CaMKII responsible for maintaining LTP? Experiments addressing the 373 role of constitutive CaMKII in the maintenance of LTP are constrained by the 374 length of recording; approximately 1 hour for whole cell and a few hours for field 375 potentials. However, if LTP is the substrate for memory, it should leave a lasting 376 memory trace at synapses, acquired while the animal is alive (Lisman, 2017; 377 Sanhueza and Lisman, 2013). A number of studies have reported a lasting 378 depression following transient inhibition of CaMKII (Barcomb et al., 2016; Gouet 379 et al., 2012; Sanhueza et al., 2011; Sanhueza et al., 2007). Furthermore, even 380 under basal conditions, CaMKII is found in isolated PSDs (Petersen et al., 2003; 381 Strack et al., 1997a) and synaptic puncta (Bayer et al., 2006) and this is in the 382 autophosphorylated state (Strack et al., 1997b; Trinidad et al., 2005). As 383 discussed above all three inhibitory peptides (myr-CN27, myr-AIP and paAIP2) 384 depress synaptic transmission and this depression requires the presence of 385 CaMKII. A number of trivial explanations, such as ongoing constitutive $\mathrm{Ca}^{2+}$ 386 activation of the enzyme, were excluded.

388 We then focused on the possibility that this constitutive action of CaMKII reflects 389 prior LTP. First, the finding that the inhibition does not affect NMDAR responses 390 is consistent with an LTP mechanism, since LTP preferentially enhances AMPAR 391 responses (reviewed in (Nicoll, 2017)). Second, there should be no recovery from 392 the transient inhibition of CaMKII, analogous to the resetting of a molecular 393 switch. This is, indeed, the case. Third, cells in which the NMDAR has been 394 deleted embryonically should be devoid of the constitutive activity. Again, this is 395 the case. This is unlikely to be due to the loss of GluN2B subunit, because cells 396 expressing a pore dead NMDAR mutant, in which assembles with GluN2B, had 397 little CaMKII constitutive activity. This finding suggests that non-NMDAR sources 398 of $\mathrm{Ca}^{2+}$ in the behaving animal are incapable of triggering CaMKII-dependent 399 enhancement in synaptic transmission. This tight linking of NMDAR sources of $400 \mathrm{Ca}^{2+}$ to CaMKII is critical, because it prevents the degrading of the Hebbian 401 nature of plasticity. Finally, it is well established that LTP is saturable. Thus, if 
402 constitutively action CaMKII represents LTP, removing this component should 403 allow for larger LTP. We find that in cells in which CaMKII is transiently blocked 404 LTP is approximately twice as large as in neighboring control cells. Taken 405 together these results suggest that constitutive CaMKII represents LTP acquired 406 at synapses while the animal was alive, thus supporting a role for CaMKII in 407 synaptic memory.

409 Based on the present results the term "basal synaptic transmission" needs to be 410 reevaluated. It has generally been assumed that the synapses studied in a 411 hippocampal slice, in which much of the afferent drive from multiple inputs have 412 been removed in the slicing, are at a ground "basal" state. The present results 413 indicate that the synaptic currents we measure are actually maintained by a 414 persistent enhancement acquired prior to slicing.

416 It should be pointed out that the contribution of CaMKII to synaptic transmission

417 is unlikely to be a simple addition to the overall preexisting excitatory drive onto 418 the cell. It has long been argued that such a scenario would be unstable and 419 quickly saturate (Bienenstock et al., 1982; Cooper and Bear, 2012; Fusi et al., 420 2005; Morrison et al., 2008; Toyoizumi et al., 2014; Turrigiano, 2008). A number 421 of well-established mechanisms exist to maintain stability, including 422 heterosynaptic depression (Scanziani et al., 1996), NMDAR-dependent long-term 423 depression (Malenka and Bear, 2004) and on a longer time scale cell wide 424 homeostasis (Turrigiano, 2008). Thus, it is envisaged that the CaMKII memory 425 trace is embedded in a network of synapses with no overall net change in the 426 excitatory drive onto the cell or network.

428 It is striking to compare the action of paAIP2 on LTP induction (Murakoshi et al., 429 2017) and on synaptic transmission (present study). A brief light exposure ( 1 430 min.), delivered immediately inducing LTP, is sufficient to prevent LTP 431 (Murakoshi et al., 2017). This finding indicates that the blocking of CaMKII 432 activation by paAIP2 is very rapid. In contrast, the effect on synaptic transmission 
433 and the reversal of LTP required 10's of minutes. What might explain the 434 dramatic difference in the kinetics of inhibition of LTP induction compared to LTP 435 maintenance and constitutive CaMKII? There is a well-accepted sequence of 436 events for the role of CaMKII in the induction of LTP (Coultrap and Bayer, 2012; 437 Hell, 2014; Lisman et al., 2012; Nicoll, 2017). Following NMDAR activation $\mathrm{Ca}^{2+}$ 438 binds to CaM, which then binds to CaMKII activating the enzyme. This causes 439 the autophosphorylation of T286 and the translocation of cytosolic CaMKII to the 440 PSD where it binds to the C-tail of GluN2B. It seems reasonable to expect that 441 under baseline conditions much of CaMKII and paAIP2 are freely diffusible in the 442 spine cytoplasm, as are phosphatases. The activation of paAIP2 would quickly 443 inhibit CaMKII autophosphorylation and its recruitment to the PSD and 444 phosphatases would quickly reverse its effect. During baseline transmission and 445 during the maintenance of LTP a fraction of CaMKII is bound to GluN2B. 446 Evidence suggests that the CaMKII/GluN2B complex is protected from 447 phosphatases (Cheriyan et al., 2011; Lisman and Raghavachari, 2015; 448 Mullasseril et al., 2007). Thus, reversing action of this sequestered CaMKII would 449 be more difficult than preventing phosphorylation of cytosolic CaMKII.

451 The present results could shed light on the underlying mechanism of long-term 452 depression (LTD). Although this topic remains contentious (Collingridge et al., 453 2010; Dore et al., 2016; Goodell et al., 2017; Lisman and Zhabotinsky, 2001; 454 Malenka and Bear, 2004; Stein et al., 2021; Wong and Gray, 2018), the long-held 455 view is that LTD is mediated by the activation of phosphatases, in particular 456 protein phosphatase 1 (PP1) (Mulkey et al., 1994; Mulkey et al., 1993). One of 457 the limitations to this model is the lack of evidence that synaptic transmission is 458 maintained by kinase activity. Our demonstration that CaMKII maintains synaptic 459 transmission provides a very simple model for LTD., i.e., a depotentiation or 460 reversal of this prior LTP. One of the features of LTD is that it takes many 461 minutes for its induction. Our finding that it takes many minutes for CaMKII 462 inhibitors to depress synaptic transmission provides an explanation for this 463 property. A formal model for the interplay between CaMKII and PP1 was 
464 proposed some time ago (Lisman and Zhabotinsky, 2001). The absence of LTD 465 in CaMKII knockout mice (Coultrap et al., 2014; Stevens et al., 1994) support 466 such a model, but does not rule out other models.

468 What might account for the failure of previous studies to detect a component of 469 synaptic transmission driven by constitutive CaMKII (Achterberg et al., 2014;

470 Buard et al., 2010; Chen et al., 2001; Feng, 1995; Giese et al., 1998; Malinow et 471 al., 1989; Murakoshi et al., 2017; Otmakhov et al., 1997; Silva et al., 1992; Wang 472 and Kelly, 1996), but see (Hinds et al., 1998), or to detect a role of constitutive 473 CaMKII in maintaining LTP (Buard et al., 2010; Chen et al., 2001; Malinow et al., 474 1989; Murakoshi et al., 2017; Otmakhov et al., 1997), but see (Feng, 1995)? We 475 suggest three factors. The first concerns the duration of peptide application, 476 which in our hands takes 10's of minutes to act, especially with field potential 477 recording. The second concerns the concentration and time. As discussed above 478 it is more difficult to reverse the constitutive action of CaMKII, than it is to block 479 its activation. Finally, the membrane permeabilizing agent is important. In our 480 hands myristoylation was more effective and specific than the cell-penetrating 481 peptide, tat.

483 In summary our results overcome many of the obstacles that have prevented 484 embracing CaMKII as a molecular storage devise. Specifically, our results show 485 that transient inhibition of CaMKII results in a lasting depression of synaptic 486 transmission with properties consistent with the erasure of prior LTP acquired 487 while the animal was alive. Furthermore, the finding that CaMKII inhibition after 488 the induction of LTP reverses LTP, establishes its role in LTP maintenance. 489 Thus, these physiological results compliment the rich biochemical literature on 490 CaMKII, making CaMKII a particularly attractive molecular storage devise. It is 491 important to note, that our results have focused on LTP and it remains open as to 492 whether CaMKII actually stores memories. Recent experiments (Rossetti et al., 493 2017) using the viral expression of the catalytically dead CaMKII K42M mutant, 494 presumed to act in a dominant negative manner, support its role in memory. 


\section{METHODS}

\section{Animals}

499

All the experimental procedures on animals were approved by the UCSF Animal Care and Use Committee, BUA \# BU002466-04C. For acute slice recordings, typically we use 5-10 animals to obtain complete dataset; for culture slice

502 recordings, we usually used 5-7 animals to obtain complete dataset.

503

\section{Experimental constructs and chemical agents}

505 The paAIP2 plasmid was obtained from Dr. Ryohei Yasuda and had been 506 characterized (Murakoshi et al., 2017). The CRISPR construct targeting at 507 CaMKIla was previously characterized (Incontro et al., 2018). For biolistic 508 experiments, all the plasmids were expressed in pCAGGS vector, which contains 509 an internal ribosome entry site (IRES) followed by the fluorophore GFP. myr510 CaMKIINtide (myr-CN27) and myr-AIP were purchased from Calbiochem. Inc

511 (catalog\#, 208921; catalog\#, 189482). MK-801 maleate was purchased from 512 HelloBio. Inc (catalog\#, HB30004). Myr-CN21 was custom ordered from Elim 513 Biopharm, Inc by myristoylating the $\mathrm{N}$-terminal of CN21:

514 KRPPKLGQIGRSKRVVIEDDR amino acid sequence.

\section{Slice culture and biolistic transfection}

518 Hippocampal cultured slices are obtained from 6-8 days old rats (Stoppini et al., 519 1991). Biolistic transfection was done 1 day after sectioning, by using a Helio 520 Gene Gun with $1 \mu \mathrm{m}$ DNA-coated gold particles (Bio-Rad). Slices were 521 maintained at $34^{\circ} \mathrm{C}$ and the medium was changed every two days. Typically,

522 slices are used for electrophysiological recording 6-8 days after transfection, 523 except for CRISPR experiments, in which slices are maintained for another week 524 before recording. 


\section{In utero electroporation}

527 E15.5 pregnant mice were anesthetized with $2.5 \%$ isoflurane in $\mathrm{O}_{2}$ and injected

528 with buprenorphine for analgesia. The lateral ventricles of embryos were injected

529 with $1 \mu \mathrm{l}$ mix plasmid DNA $(1 \mu \mathrm{g} / \mu \mathrm{l})$ with beveled micropipette. Each embryo was

530 electoporated with $5 \times 50 \mathrm{~ms}, 35-40 \mathrm{~V}$ pulse.

531

532

533

\section{Acute slice preparation}

534 Mice of 2-3 weeks of age were anesthetized with $4 \%$ isoflurane, decapitated, and

535 the brain dissected free. The whole brain was sliced into $300 \mu \mathrm{m}$ slices in cutting

536 solution as described (Granger et al., 2013); recovery at $34{ }^{\circ} \mathrm{C}$ for half an hour

537 and then stored at room temperature. Solutions were continuously gassed with

$53895 \% \mathrm{O}_{2} / 5 \% \mathrm{CO}_{2}$.

\section{Photostimulation}

540 Blue light pulses $(0.1 \mathrm{~Hz}, 1 \mathrm{~s}, 20 \mathrm{~mW} / \mathrm{cm} 2)$ were generated by $473 \mathrm{~nm}$ blue DPSS

541 laser (Shanghai Laser\&Optics Century, BL473T8-300FC). The blue light from a

542 laser was delivered through a optical patch cable connected to the optical fibers.

543 Light pulses were controlled by a Master-8 (A. M. P. I). The blue light is applied

544 for 1 s duration with interval of 10 s.

545

\section{Injection of MK-801}

547 MK-801 was dissolved in saline solution and administered as a single injection i.p.

548 (10 mg/kg body weight) one hour before brain slicing.

549

550 Electrophysiological recording

551 Whole-cell voltage clamp recordings were obtained from either wild type cells or

552 fluorescent transfected pyramidal cell in CA1 region of hippocampus (Schnell et

553 al., 2002). Where indicated dual recordings were made from control and

554 transfected cells. Pyramidal neurons were identified by location and morphology.

555 All recordings were made at $20-25^{\circ} \mathrm{C}$. Internal solution (in mM): $135 \mathrm{CsMeSO}_{4}, 8$

$556 \mathrm{NaCl}, 10$ HEPES, 5 QX314-Cl, 4 Mg-ATP, 0.3 Na-GTP, 0.3 EGTA, 0.1 spermine. 
557 Osmolarity was adjusted to $290-295 \mathrm{mOm}$ and $\mathrm{pH}$ was buffered at 7.3-7.4.

558 External solution (mM): $119 \mathrm{NaCl}, 2.5 \mathrm{KCl}, 4 \mathrm{CaCl}_{2}, 4 \mathrm{MgCl}_{2}, 1 \mathrm{NaH}_{2} \mathrm{PO}_{4}, 26.2$

$559 \mathrm{NaHCO}_{3}, 11$ Glucose, bubbled continuously with $95 \% \mathrm{O}_{2} / 5 \% \mathrm{CO}_{2}$. For field

560 recordings, the internal solution was $3 \mathrm{M} \mathrm{NaCl}$ with a large opening pipette tip.

561 Synaptic currents were evoked every $10 \mathrm{~s}$ with bipolar stimulating electrodes

562 placed in s. radiatum. To record EPSCs, picrotoxin $(100 \mu \mathrm{M})$ was added to the

563 external solution; for recording of AMPAR EPSCs, the cell membrane was held

564 at $-70 \mathrm{mV}$, while for NMDAR EPSCs, it was hold at $+40 \mathrm{mV}$; LTP are induced by

565 stimulating at $2 \mathrm{~Hz}$ for 90 seconds while clamping the cell at $0 \mathrm{mV}$. For two

566 independent synaptic pathway experiments, two bipolar stimulating electrodes

567 were positioned to each side of the recording electrode with a distance of around

$568100 \mu \mathrm{m}$ and alternately stimulated every $20 \mathrm{~s}$. Current responses were collected

569 with a Multiclamp 700B amplifier (Axon Instruments), filtered at $2 \mathrm{kHz}$, and

570 digitized at $10 \mathrm{kHz}$. Cells with series resistance larger than $20 \mathrm{MOhm}$ were

571 excluded from analysis.

572

573 Statistical analysis

574 Data analysis was carried out in Igor Pro (Wavemetrics), Excel (Microsoft), and

575 GraphPad Prism (GraphPad Software). All paired recording data were analyzed

576 statistically with a Wilcoxon Signed Rank Test for paired data. For unpaired data,

577 a Mann-Whitney $U$ test was used. Statistical parameters including the types of

578 the statistical tests used, exact value of $n$, precision measures (mean \pm SEM)

579 and statistical significance are reported in the figure legends. All statistical tests 580 performed were two-sided, and with all tests a p-value of $<0.05$ was considered

581 statistically significant. All error bars represent standard error of the mean.

582

583 ACKNOWLEDGMENTS

584 
585 We thank Dr. R. Yasuda for the paAIP2 plasmids, Dr. Wei Lu for contributing the 586 data for Fig. 3A and Dr. Lonnie Wullmuth for GluN1 pore dead plasmid 587 (GluN1(N812W)). We thank members of the Nicoll lab for comments on the 588 manuscript. We appreciate the technical assistance from Eric Dang. This 589 research was supported by grant $\mathrm{MH} 070957$.

590

591

592

593 Figure legends

594

595 Figure 1. CaMKII inhibitor myr-CN27 inhibits AMPAR synaptic transmission 596 through CaMKIla. (A) Time course of myr-CN27 $(1 \mu \mathrm{M})$ inhibition on AMPAR 597 EPSCs and NMDAR EPSCs in acute slices. (B) Summary graphs showing myr598 CN27 inhibited AMPAR EPSCs (before drug: $63.9 \pm 12$ pA; after drug: $27.6 \pm 3.9$ $599 \mathrm{pA} ; \mathrm{n}=10, \mathrm{p}<0.01$, two-tailed Wilcoxon Signed Rank Test), but not NMDAR 600 EPSCs (before drug: $43.2 \pm 13.7 \mathrm{pA}$; after drug: $42 \pm 13.4 \mathrm{pA} ; \mathrm{n}=5, \mathrm{p}>0.05$, 601 two-tailed Wilcoxon Signed Rank Test). (C) Time course of the effect of myr602 CN27 on AMPAR EPSCs in wt cells and simultaneously recorded CRISPR603 CaMKIla transfected cells, normalized to wt baseline (from culture slices). While 604 myr-CN27 inhibited AMPAR EPSCs in wt cells, it had no effect on CRISPR605 CaMKIla transfected cells $(n=6 p>0.05$, two-tailed Wilcoxon Signed Rank 606 Test). (D) Sample traces showing that myr-CN27 inhibited AMPAR EPSCs in 607 control cells, but had no effect in CRISPR- CaMKIla transfected cells. Black 608 traces are control cell, green traces are transfected cell. Mean \pm s.e.m. ${ }^{* \star} P<$ 609 0.01. (E) A comparison of the reduction of synaptic transmission in field and 610 whole cell recording. For the field recording (black, $n=8$ ) after a 60 min stable 611 baseline, $1 \mu \mathrm{M}$ myr-CN27 was applied to the slice for $100 \mathrm{~min}$. For the whole cell 612 recording (blue, $\mathrm{n}=7$ ), the drug was applied after a 10 min stable baseline. In 613 both, the response was reduced to $50 \%$ of baseline. Example traces for both are 614 shown taken at the timepoints indicated by 1 and 2. The field EPSPs shown on 615 the bottom right have scale bars vertical $0.1 \mathrm{mV}$ and $5 \mathrm{~ms}$ horizontal. The whole 
616 cell recording shown bottom left have scale bars $50 \mathrm{pA}$ vertical and $20 \mathrm{~ms}$ 617 horizontal. Both recordings are done in WT slices with the whole cell recordings 618 done in slice culture DIV3-14 while the fields were done in acute slices from P1561928 mice.

621 Figure 2. CaMKII inhibitor AIP inhibits AMPAR synaptic transmission 622 through CaMKIla. (A) In culture slices, transfection of AIP reduced AMPAR 623 EPSCs (left, $n=10, p<0.01$, two-tailed Wilcoxon Signed Rank Test) but not 624 NMDAR EPSCs (right, $n=10, p>0.05$, two-tailed Wilcoxon Signed Rank Test). 625 Sample traces show the effects of AIP on AMPAR EPSCs and NMDAR EPSCs.

626 (B) AMPA/NMDA ratios compared to WT $(n=34)$ are reduced after myr-AIP (20 $627 \mu \mathrm{M})$ treatment $(n=12)$. Scale bar $=50 \mathrm{pA}$ vertical and $30 \mathrm{~ms}$ horizontal. $(\mathbf{C})$ Left, 628 Summary of the effect of myr-AIP $(20 \mu \mathrm{M})$ on AMPAR EPSCs in wt cells $(n=6)$ 629 and interleaved CRISPR-CaMKIla transfected cells $(n=5)$ from culture slices, 630 normalized to each cell's baseline. The difference between control and CRISPR631 CaMKIla transfected cells at 30 min: $p<0.01$, Mann-Whitney $U$ test. Right, 632 sample traces showing myr-AIP inhibition of AMPAR EPSC in control cells, but 633 not in CRISPR- CaMKIla transfected cells. Black traces are control cell, green 634 are transfected cell. Mean \pm s.e.m.

636 Figure 3. Light-activated CaMKII inhibitor paAIP2 inhibits AMPAR synaptic 637 transmission through CaMKIla. (A) Time course of paAIP2 effect on AMPAR 638 and NMDAR synaptic transmission in culture slices. Blue light exposure inhibited 639 AMPAR synaptic transmission in paAIP2 expressing cells (green circles), but not 640 in simultaneously recorded control cells (black circles) or NMDAR synaptic 641 transmission (red circles). (B) Summary data showing that paAIP2 inhibited 642 AMPAR synaptic transmission (before light: $51 \pm 13.8 \mathrm{pA}$; after light: $26.5 \pm 7.2$ $643 \mathrm{pA} ; \mathrm{n}=12, \mathrm{p}<0.01$, two-tailed Wilcoxon Signed Rank Test), but had no effect on 644 NMDAR synaptic transmission (before light: $31.2 \pm 7.6$ pA; after light: $29.8 \pm 7.7$ 
$645 \mathrm{pA} ; \mathrm{n}=5, \mathrm{p}>0.05$, two-tailed Wilcoxon Signed Rank Test). Mean \pm s.e.m. ${ }^{*} P<$ 6460.01.

648 Figure 4. The depression caused by transient inhibition of CaMKII is long 649 lasting and is absent in cells lacking NMDARs. (A) In acute slices, time 650 course showing that following myr-CN27 application AMPAR synaptic 651 transmission remains depressed (The difference between before and after myr652 CN27: $n=4, p<0.01$, two-tailed Wilcoxon Signed Rank Test). (B) In culture 653 slices, time course showing that following blue light exposure on paAIP2 654 expressing cells AMPAR synaptic transmission remains depressed (The 655 difference between before and after blue light exposure: $n=5, p<0.01$, two656 tailed Wilcoxon Signed Rank Test). (C) Left panel, paired recordings from acute 657 slices of control cells and those expressing Cre in GluN1 floxed mice ( $n=5, p<$ 658 0.01, two-tailed Wilcoxon Signed Rank Test); Middle panel, time course of the 659 myr-AIP effect on AMPAR synaptic transmission in control cells $(n=5)$ and 660 interleaved cells expressing Cre in GluN1 floxed mice $(n=5) . p<0.01$, Mann661 Whitney $U$ test. Right panel, sample traces showing myr-AIP inhibited AMPAR 662 synaptic transmission in control cell (black traces), but not in a GluN1KO cell 663 (green traces). Mean \pm s.e.m. (D) Left panel, paired recordings from acute slices 664 of control cells and those expressing Cre and N812W (pore dead, PD) in GluN1 665 floxed mice ( $n=6, p<0.05$, two-tailed Wilcoxon Signed Rank Test); Middle panel, 666 the action of Myr-CN27 is not rescued by expressing a GluN1 pore dead mutant. $667 \mathrm{n}=8$; Right panel, sample traces showing myr-CN27 inhibited AMPAR synaptic 668 transmission in control cell (black traces), but not in a GluN1KO+ PD cell (green 669 traces).

671 Figure 5. Transient inhibition of CaMKII enhances subsequent LTP. (A) 672 Timeline of experimental procedure. Following in utero electroporation acute 673 slices were prepared at P15-P25. Slices were then exposed to blue light for 40 674 minutes. Paired recordings were then made from a control cell (black circles) and 675 a paAIP2 expressing cell (green circles). (B) One representative experiment 
676 showing that prior paAIP2 activation reduced baseline AMPAR EPSCs, but LTP 677 was larger than that recorded in the neighboring control cell. (C) Normalized 678 representative experiment showing that prior paAIP2 activation increased LTP.

679 (D) Summary data showing that prior paAIP2 activation reduced baseline 680 AMPAR EPSCs, but enhanced LTP $(n=5)$. (E) Normalized summary data 681 showing that prior paAIP2 activation doubles the size of LTP. $n=5, p<0.01$, 682 two-tailed Wilcoxon Signed Rank Test. Mean \pm s.e.m.

684 Figure 6. Myr-CN27 erases LTP in acute slices. (A) cartoon diagram of two 685 pathway experiment. To record the response from two independent pathways, 686 two bipolar stimulating electrodes were positioned to either side of the recorded 687 cell with a distance of around $100 \mu \mathrm{m}$. Stimuli were applied alternately every $20 \mathrm{~s}$. 688 (B) A sample experiment showing that myr-CN27 inhibited the LTP pathway 689 more strongly than the control pathway. (C) Left, summary data showing that myr-CN27 reduced the control pathway (black circles) 50\%, while completely 691 reversing LTP (red circles) (The difference between control and LTP pathway at 69260 min: $n=11, p>0.05$, two-tailed Wilcoxon Signed Rank Test). Responses are 693 mean \pm s.e.m. Right, sample traces showing the effect of myr-CN27 on AMPA 694 EPSCs in control and LTP pathway. LTP is induced by $2 \mathrm{~Hz}$ stimulation for $90 \mathrm{~s}$, 695 while holding the cell at $0 \mathrm{mV}$.

\section{Supplemental fig. legends}

Figure S1/ Figure 1-figure suple1. 700 inhibitors on the AMPAR/NMDAR ratio AMPAR/NMDAR ratios recorded in acute slices made from WT mice incubated without inhibitor treatment (WT) (n

$702=34)$, with $5 \mu \mathrm{M}$ tatCN21 ( $=10)$, with $1 \mu \mathrm{M}$ myr-CN27 $(\mathrm{n}=47)$, and with $5 \mu \mathrm{M}$ 703 myr-CN21 $(n=9)$. Sample traces are shown at top. 704 and 30 ms horizontal $p<0.01$, Mann-Whitney $U$ test. 
705 Figure S2/Figure 3-figure suple1. Characterization of synaptic transmission

706

707

708

709

710

711

712

713

714

715

716

717

718

719

720

721

722

723

724

725

726

727

728

729

730

731

732

733

734 in control cells and paAIP2 transfected cells from culture slices, before and after blue light exposure. (A, B) transfection of paAIP2 had no effect on AMPAR synaptic transmission (control, $96.4 \pm 19.7$ pA; paAIP2, $96.1 \pm 21.3$ pA; $\mathrm{n}=16, \mathrm{p}>0.05$, two-tailed Wilcoxon Signed Rank Test), or NMDAR synaptic transmission (control, 81.5 $\pm 10.1 \mathrm{pA}$; paAIP2, $89.7 \pm 16.6 \mathrm{pA} ; \mathrm{n}=11, \mathrm{p}>0.05$, two-tailed Wilcoxon Signed Rank Test). (C, D) blue light exposure reduced AMPAR synaptic transmission in paAIP2 transfected cells (control, 69.1 \pm 11.1 pA; paAIP2, $31.7 \pm 7.1 \mathrm{pA} ; \mathrm{n}=8, \mathrm{p}<0.01$, two-tailed Wilcoxon Signed Rank Test), but have no effect on NMDAR synaptic transmission (control, $86.5 \pm 23.7$ pA; paAIP2, $78 \pm 20.6$ pA; $n=8, p>0.05$, two-tailed Wilcoxon Signed Rank Test). Mean \pm s.e.m.

Figure S3/ Figure 3-figure suple2. CRISPR deletion of CaMKIla prevents paAIP2 effect on AMPAR synaptic transmission in culture slices. Blue light exposure on transfected cells (with co-expression of paAIP2 and CRISPRCaMKIlla) did not inhibit AMPAR EPSCs $(n=5, p>0.05$, two-tailed Wilcoxon Signed Rank Test). Mean \pm s.e.m.

Figure S4/ Figure 4-figure suple1. Origin of constitutive CaMKIl effect on synaptic transmission in acute slices. (A) Loading cells with $15 \mathrm{mM}$ BAPTA did not alter the CN-27 induced depression of AMPAR synaptic transmission ( $\mathrm{n}=$ $5, p<0.01$, two-tailed Wilcoxon Signed Rank Test). (B) Preinjection of MK801 (i.p.10 mg/kg body weight) one hour before slicing greatly depressed the NMDAR EPSC resulting in a large increase in the AMPAR/NMDAR ratio (control: $0.72 \pm$ $0.11, n=5$; MK-801: $4.55 \pm 0.58, n=5 ; p<0.01$, Mann-Whitney $U$ test). (C) With preinjection of MK-801 one hour before slicing, and in the presence of MK-801 $(100 \mu \mathrm{M})$, myr-CN27 inhibited AMPAR synaptic transmission $(\mathrm{n}=5, \mathrm{p}<0.01$, two-tailed Wilcoxon Signed Rank Test). 
735 Figure S5/ Figure 6-figure suple1. Reversal of LTP and reduction of 736 synaptic transmission with application of $1 \mu \mathrm{M}$ myr-CN27. After a $55 \mathrm{~min}$ 737 stable baseline in both pathways (Control = Black, LTP $=$ Red, both $n=1$ ), a train 738 of $100 \mathrm{~Hz}$ for $1 \mathrm{~s}$ stimuli was applied 4 times with an interval of $20 \mathrm{~s}$. Following 739 the stimulus, the LTP pathway exhibited a rapid increase in fEPSP slope that 740 plateaued at $\sim 140 \%$ of baseline. The control pathway did not exhibit this 741 increase. After a stable baseline was achieved for the LTP pathway, $1 \mu \mathrm{M}$ myr-

742 CN27 was applied. Following application, both pathways exhibited a decrease in 743 fEPSP slope with the LTP pathway exhibiting a greater decrease. Example 744 traces for both pathways are displayed below the graph and taken at the 745 indicated timepoints $(1,2,3)$. Traces are colored according to the pathway they 746 correspond to. The scale bars are vertical $0.1 \mathrm{mV}$ and $5 \mathrm{~ms}$ horizontal. Recording 747 done in WT acute slices from P15-28 mice.

750 Figure1-Source Data File1. CaMKII inhibitor myr-CN27 inhibits AMPAR 751 synaptic transmission through CaMKIla. In this dataset, the results of the 752 effects of myr-CN27 on AMPAR EPSCs and NMDAR EPSCs are included.

753 Figure2-Source Data File1. CaMKII inhibitor AIP inhibits AMPAR synaptic 754 transmission through CaMKIla. In this dataset, the results of the effects of AIP 755 on AMPAR EPSCs and NMDAR EPSCs are included.

756 Figure3-Source Data File1. Light-activated CaMKII inhibitor paAIP2 inhibits 757 AMPAR synaptic transmission through CaMKIla. In this dataset, the results of

758 the effects of paAIP2 on AMPAR EPSCs and NMDAR EPSCs are included.

759

760 Figure 4-Source Data File1. The depression caused by transient inhibition 761 of CaMKII is long lasting and is absent in cells lacking NMDARs. In this 762 dataset, the results of the effects of myr-CN27 and paAIP2 on AMPAR EPSCs 763 over time are included. 
765 Figure 5-Source Data File1. Transient inhibition of CaMKII enhances subsequent LTP. In this dataset, the results of the effects of LTP induction on control cells and pre-inhibition cells are included.

Figure 6-Source Data File1. Myr-CN27 erases LTP in acute slices. In this dataset, the results of the effects of myr-CN27 on LTP and control pathways are

771 included.

772

\section{References}

774 Achterberg, K.G., Buitendijk, G.H., Kool, M.J., Goorden, S.M., Post, L., Slump, D.E., Silva, A.J., van Woerden, G.M., Kushner, S.A., and Elgersma, Y. (2014).

776

777

778

779

780

781

782

783

784

785

786

787

788 Temporal and region-specific requirements of alphaCaMKII in spatial and contextual learning. J Neurosci 34, 11180-11187.

Allen, J.K., Brock, D.J., Kondow-McConaghy, H.M., and Pellois, J.P. (2018). Efficient Delivery of Macromolecules into Human Cells by Improving the Endosomal Escape Activity of Cell-Penetrating Peptides: Lessons Learned from dfTAT and its Analogs. Biomolecules 8.

Amin, J.B., Salussolia, C.L., Chan, K., Regan, M.C., Dai, J., Zhou, H.X., Furukawa, H., Bowen, M.E., and Wollmuth, L.P. (2017). Divergent roles of a peripheral transmembrane segment in AMPA and NMDA receptors. J Gen Physiol 149, 661-680.

Barcomb, K., Hell, J.W., Benke, T.A., and Bayer, K.U. (2016). The CaMKII/GluN2B Protein Interaction Maintains Synaptic Strength. J Biol Chem 291, 16082-16089.

789 Bayer, K.U., De Koninck, P., Leonard, A.S., Hell, J.W., and Schulman, H. (2001).

790 Interaction with the NMDA receptor locks CaMKII in an active conformation.

791 Nature 411, 801-805.

792

793

794

795

796

797

798

799

800

801

802

Bayer, K.U., LeBel, E., McDonald, G.L., O'Leary, H., Schulman, H., and De Koninck, P. (2006). Transition from reversible to persistent binding of CaMKII to postsynaptic sites and NR2B. J Neurosci 26, 1164-1174.

Bayer, K.U., and Schulman, H. (2019). CaM Kinase: Still Inspiring at 40. Neuron 103, 380-394.

Benfenati, F., Valtorta, F., Rubenstein, J.L., Gorelick, F.S., Greengard, P., and Czernik, A.J. (1992). Synaptic vesicle-associated Ca2+/calmodulin-dependent protein kinase II is a binding protein for synapsin I. Nature 359, 417-420.

Bhattacharyya, M., Karandur, D., and Kuriyan, J. (2020). Structural Insights into

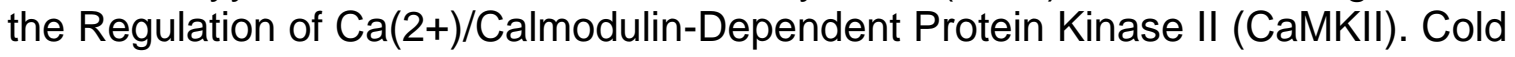
Spring Harb Perspect Biol 12.

803 Bhattacharyya, M., Stratton, M.M., Going, C.C., McSpadden, E.D., Huang, Y., 804 Susa, A.C., Elleman, A., Cao, Y.M., Pappireddi, N., Burkhardt, P., et al. (2016). 
805

806

807

808

809

810

811

812

813

814

815

816

817

818

819

820

821

822

823

824

825

826

827

828

829

830

831

832

833

834

835

836

837

838

839

840

841

842

843

844

845

846

847

848

849

Molecular mechanism of activation-triggered subunit exchange in $\mathrm{Ca}(2+) /$ calmodulin-dependent protein kinase II. Elife 5 .

Bienenstock, E.L., Cooper, L.N., and Munro, P.W. (1982). Theory for the development of neuron selectivity: orientation specificity and binocular interaction in visual cortex. J Neurosci 2, 32-48.

Bird, T.D., and Plum, F. (1968). Recovery from barbiturate overdose coma with a prolonged isoelectric electroencephalogram. Neurology 18, 456-460.

Buard, I., Coultrap, S.J., Freund, R.K., Lee, Y.S., Dell'Acqua, M.L., Silva, A.J., and Bayer, K.U. (2010). CaMKII "autonomy" is required for initiating but not for maintaining neuronal long-term information storage. J Neurosci 30, 8214-8220.

Cajal, S.R. (1911). Histologie du Systeme Nerveux de l'Homme et des Vertebres. Translated by N. Swanson and L. W. Swanson, Oxford University Press, New York.

Chang, B.H., Mukherji, S., and Soderling, T.R. (1998). Characterization of a calmodulin kinase II inhibitor protein in brain. Proc Natl Acad Sci U S A 95, 10890-10895.

Chang, B.H., Mukherji, S., and Soderling, T.R. (2001). Calcium/calmodulindependent protein kinase II inhibitor protein: localization of isoforms in rat brain. Neuroscience 102, 767-777.

Chen, H.X., Otmakhov, N., Strack, S., Colbran, R.J., and Lisman, J.E. (2001). Is persistent activity of calcium/calmodulin-dependent kinase required for the maintenance of LTP? J Neurophysiol 85, 1368-1376.

Cheriyan, J., Kumar, P., Mayadevi, M., Surolia, A., and Omkumar, R.V. (2011). Calcium/calmodulin dependent protein kinase II bound to NMDA receptor 2B subunit exhibits increased ATP affinity and attenuated dephosphorylation. PLoS One 6, e16495.

Choquet, D. (2018). Linking Nanoscale Dynamics of AMPA Receptor Organization to Plasticity of Excitatory Synapses and Learning. J Neurosci 38, 9318-9329.

Collingridge, G.L., Isaac, J.T., and Wang, Y.T. (2004). Receptor trafficking and synaptic plasticity. Nat Rev Neurosci 5, 952-962.

Collingridge, G.L., Peineau, S., Howland, J.G., and Wang, Y.T. (2010). Longterm depression in the CNS. Nat Rev Neurosci 11, 459-473.

Cooper, L.N., and Bear, M.F. (2012). The BCM theory of synapse modification at 30: interaction of theory with experiment. Nat Rev Neurosci 13,798-810.

Coultrap, S.J., and Bayer, K.U. (2012). CaMKII regulation in information processing and storage. Trends Neurosci 35, 607-618.

Coultrap, S.J., Freund, R.K., O'Leary, H., Sanderson, J.L., Roche, K.W., Dell'Acqua, M.L., and Bayer, K.U. (2014). Autonomous CaMKII mediates both LTP and LTD using a mechanism for differential substrate site selection. Cell Rep 6, 431-437.

D'Esposito, M., and Postle, B.R. (2015). The cognitive neuroscience of working memory. Annu Rev Psychol 66, 115-142.

Davies, S.N., Martin, D., Millar, J.D., Aram, J.A., Church, J., and Lodge, D. (1988). Differences in results from in vivo and in vitro studies on the use- 
850 dependency of $\mathrm{N}$-methylaspartate antagonism by MK-801 and other 851 phencyclidine receptor ligands. Eur J Pharmacol 145, 141-151.

852 Dore, K., Aow, J., and Malinow, R. (2016). The Emergence of NMDA Receptor 853 Metabotropic Function: Insights from Imaging. Front Synaptic Neurosci 8, 20.

854 Feng, T.P. (1995). The involvement of PKC and multifunctional CaM kinase II of 855 the postsynaptic neuron in induction and maintenance of long-term potentiation. 856 Prog Brain Res 105, 55-63.

857 Fusi, S., Drew, P.J., and Abbott, L.F. (2005). Cascade models of synaptically 858 stored memories. Neuron 45, 599-611.

859 Giese, K.P., Fedorov, N.B., Filipkowski, R.K., and Silva, A.J. (1998). 860 Autophosphorylation at Thr286 of the alpha calcium-calmodulin kinase II in LTP 861 and learning. Science 279, 870-873.

862 Goodell, D.J., Zaegel, V., Coultrap, S.J., Hell, J.W., and Bayer, K.U. (2017). 863 DAPK1 Mediates LTD by Making CaMKII/GluN2B Binding LTP Specific. Cell Rep 864 19, 2231-2243.

865 Goold, C.P., and Nicoll, R.A. (2010). Single-cell optogenetic excitation drives 866 homeostatic synaptic depression. Neuron 68, 512-528.

867 Gouet, C., Aburto, B., Vergara, C., and Sanhueza, M. (2012). On the mechanism 868 of synaptic depression induced by CaMKIIN, an endogenous inhibitor of CaMKII. 869 PLoS One 7, e49293.

870 Granger, A.J., Shi, Y., Lu, W., Cerpas, M., and Nicoll, R.A. (2013). LTP requires 871 a reserve pool of glutamate receptors independent of subunit type. Nature 493, 872 495-500.

873 Hell, J.W. (2014). CaMKII: claiming center stage in postsynaptic function and 874 organization. Neuron 81, 249-265.

875 Hinds, H.L., Goussakov, I., Nakazawa, K., Tonegawa, S., and Bolshakov, V.Y. 876 (2003). Essential function of alpha-calcium/calmodulin-dependent protein kinase 877 II in neurotransmitter release at a glutamatergic central synapse. Proc Natl Acad 878 Sci U S A 100, 4275-4280.

879 Hinds, H.L., Tonegawa, S., and Malinow, R. (1998). CA1 long-term potentiation 880 is diminished but present in hippocampal slices from alpha-CaMKII mutant mice. 881 Learn Mem 5, 344-354.

882 Huganir, R.L., and Nicoll, R.A. (2013). AMPARs and synaptic plasticity: the last 88325 years. Neuron 80, 704-717.

884 Inagaki, H.K., Fontolan, L., Romani, S., and Svoboda, K. (2019). Discrete 885 attractor dynamics underlies persistent activity in the frontal cortex. Nature 566, 886 212-217.

887 Incontro, S., Diaz-Alonso, J., lafrati, J., Vieira, M., Asensio, C.S., Sohal, V.S., 888 Roche, K.W., Bender, K.J., and Nicoll, R.A. (2018). The CaMKII/NMDA receptor 889 complex controls hippocampal synaptic transmission by kinase-dependent and 890 independent mechanisms. Nat Commun 9, 2069.

891 Ishida, A., Shigeri, Y., Tatsu, Y., Uegaki, K., Kameshita, I., Okuno, S., Kitani, T., 892 Yumoto, N., and Fujisawa, H. (1998). Critical amino acid residues of AIP, a highly 893 specific inhibitory peptide of calmodulin-dependent protein kinase II. FEBS Lett 894 427, 115-118. 
895 Kennedy, M.B. (2013). Synaptic Signaling in Learning and Memory. Cold Spring 896 Harb Perspect Biol 8, a016824.

897 LeCher, J.C., Nowak, S.J., and McMurry, J.L. (2017). Breaking in and busting 898 out: cell-penetrating peptides and the endosomal escape problem. Biomol 899 Concepts 8, 131-141.

900 Lee, S.J., Escobedo-Lozoya, Y., Szatmari, E.M., and Yasuda, R. (2009). 901 Activation of CaMKII in single dendritic spines during long-term potentiation. 902 Nature 458, 299-304.

903 Leonard, A.S., Lim, I.A., Hemsworth, D.E., Horne, M.C., and Hell, J.W. (1999). 904 Calcium/calmodulin-dependent protein kinase II is associated with the N-methyl905 906 907 908 909 910

911

912

913

914

915

916

917

918

919

920

921

922

923

924

925

926

927

928

929

930

931

932

933

934

935

936

937

938

939

940 D-aspartate receptor. Proc Natl Acad Sci U S A 96, 3239-3244.

Lisman, J. (2017). Criteria for identifying the molecular basis of the engram (CaMKII, PKMzeta). Mol Brain 10, 55.

Lisman, J., and Raghavachari, S. (2015). Biochemical principles underlying the stable maintenance of LTP by the CaMKII/NMDAR complex. Brain Res 1621, 5161.

Lisman, J., Schulman, H., and Cline, H. (2002). The molecular basis of CaMKII function in synaptic and behavioural memory. Nat Rev Neurosci 3, 175-190.

Lisman, J., Yasuda, R., and Raghavachari, S. (2012). Mechanisms of CaMKII action in long-term potentiation. Nat Rev Neurosci 13, 169-182.

Lisman, J.E., and Goldring, M.A. (1988). Feasibility of long-term storage of graded information by the $\mathrm{Ca} 2+$ /calmodulin-dependent protein kinase molecules of the postsynaptic density. Proc Natl Acad Sci U S A 85, 5320-5324.

Lisman, J.E., and Zhabotinsky, A.M. (2001). A model of synaptic memory: a CaMKII/PP1 switch that potentiates transmission by organizing an AMPA receptor anchoring assembly. Neuron 31, 191-201.

Lledo, P.M., Hjelmstad, G.O., Mukherji, S., Soderling, T.R., Malenka, R.C., and Nicoll, R.A. (1995). Calcium/calmodulin-dependent kinase II and long-term potentiation enhance synaptic transmission by the same mechanism. Proc Natl Acad Sci U S A 92, 11175-11179.

Malenka, R.C., and Bear, M.F. (2004). LTP and LTD: an embarrassment of riches. Neuron 44, 5-21.

Malenka, R.C., Kauer, J.A., Perkel, D.J., Mauk, M.D., Kelly, P.T., Nicoll, R.A., and Waxham, M.N. (1989). An essential role for postsynaptic calmodulin and protein kinase activity in long-term potentiation. Nature 340, 554-557.

Malinow, R., and Malenka, R.C. (2002). AMPA receptor trafficking and synaptic plasticity. Annu Rev Neurosci 25, 103-126.

Malinow, R., Schulman, H., and Tsien, R.W. (1989). Inhibition of postsynaptic PKC or CaMKII blocks induction but not expression of LTP. Science 245, 862866.

Maravall, M., Mainen, Z.F., Sabatini, B.L., and Svoboda, K. (2000). Estimating intracellular calcium concentrations and buffering without wavelength ratioing. Biophys J 78, 2655-2667.

Miller, S.G., and Kennedy, M.B. (1986). Regulation of brain type II $\mathrm{Ca} 2+/$ calmodulin-dependent protein kinase by autophosphorylation: a $\mathrm{Ca2+-}$ triggered molecular switch. Cell 44, 861-870. 
941 Morrison, A., Diesmann, M., and Gerstner, W. (2008). Phenomenological models 942 of synaptic plasticity based on spike timing. Biol Cybern 98, 459-478.

943 Mulkey, R.M., Endo, S., Shenolikar, S., and Malenka, R.C. (1994). Involvement

944 of a calcineurin/inhibitor-1 phosphatase cascade in hippocampal long-term

945

946

947

948

949

950

951

952

953

954

955

956

957

958

959

960

961

962

963

964

965

966

967

968

969

970

971

972

973

974

975

976

977

978

979

980

981

982

983

984

985

986 depression. Nature 369, 486-488.

Mulkey, R.M., Herron, C.E., and Malenka, R.C. (1993). An essential role for protein phosphatases in hippocampal long-term depression. Science 261, 10511055.

Mullasseril, P., Dosemeci, A., Lisman, J.E., and Griffith, L.C. (2007). A structural mechanism for maintaining the 'on-state' of the CaMKII memory switch in the post-synaptic density. J Neurochem 103, 357-364.

Murakoshi, H., Shin, M.E., Parra-Bueno, P., Szatmari, E.M., Shibata, A.C.E., and Yasuda, R. (2017). Kinetics of Endogenous CaMKII Required for Synaptic Plasticity Revealed by Optogenetic Kinase Inhibitor. Neuron 94, 690.

Nelson, A.R., Borland, L., Allbritton, N.L., and Sims, C.E. (2007). Myristoyl-based transport of peptides into living cells. Biochemistry 46, 14771-14781.

Nicoll, R.A. (2017). A Brief History of Long-Term Potentiation. Neuron 93, 281290.

Otmakhov, N., Griffith, L.C., and Lisman, J.E. (1997). Postsynaptic inhibitors of calcium/calmodulin-dependent protein kinase type II block induction but not maintenance of pairing-induced long-term potentiation. J Neurosci 17, 5357-5365. Özden, C., Santos, N., Agnello, E., Gaubitz, C., Lapinskas, E., and al., e. (2020). CaMKII binds both substrates and effectors at the active site bioRxiv: https://doiorg/101101/20201025354241.

Patel, S.G., Sayers, E.J., He, L., Narayan, R., Williams, T.L., Mills, E.M., Allemann, R.K., Luk, L.Y.P., Jones, A.T., and Tsai, Y.H. (2019). Cell-penetrating peptide sequence and modification dependent uptake and subcellular distribution of green florescent protein in different cell lines. Sci Rep 9, 6298.

Pellicena, P., and Schulman, H. (2014). CaMKII inhibitors: from research tools to therapeutic agents. Front Pharmacol 5, 21.

Petersen, J.D., Chen, X., Vinade, L., Dosemeci, A., Lisman, J.E., and Reese, T.S. (2003). Distribution of postsynaptic density (PSD)-95 and Ca2+/calmodulindependent protein kinase II at the PSD. J Neurosci 23, 11270-11278.

Pettit, D.L., Perlman, S., and Malinow, R. (1994). Potentiated transmission and prevention of further LTP by increased CaMKII activity in postsynaptic hippocampal slice neurons. Science 266, 1881-1885.

Pi, H.J., Otmakhov, N., Lemelin, D., De Koninck, P., and Lisman, J. (2010). Autonomous CaMKII can promote either long-term potentiation or long-term depression, depending on the state of T305/T306 phosphorylation. J Neurosci 30, 8704-8709.

Poncer, J.C., Esteban, J.A., and Malinow, R. (2002). Multiple mechanisms for the potentiation of AMPA receptor-mediated transmission by alpha-Ca2+/calmodulindependent protein kinase II. J Neurosci 22, 4406-4411.

Rossetti, T., Banerjee, S., Kim, C., Leubner, M., Lamar, C., Gupta, P., Lee, B., Neve, R., and Lisman, J. (2017). Memory Erasure Experiments Indicate a Critical Role of CaMKII in Memory Storage. Neuron 96, 207-216 e202. 
987 Sanhueza, M., Fernandez-Villalobos, G., Stein, I.S., Kasumova, G., Zhang, P., 988 Bayer, K.U., Otmakhov, N., Hell, J.W., and Lisman, J. (2011). Role of the 989 CaMKII/NMDA receptor complex in the maintenance of synaptic strength. J 990 Neurosci 31, 9170-9178.

991 Sanhueza, M., and Lisman, J. (2013). The CaMKII/NMDAR complex as a 992

993

994

995

996

997

998

999

1000

1001

1002

1003

1004

1005

1006

1007

1008

1009

1010

1011

1012

1013

1014

1015

1016

1017

1018

1019

1020

1021

1022

1023

1024

1025

1026

1027

1028

1029

1030

1031

1032 molecular memory. Mol Brain 6, 10.

Sanhueza, M., Mclntyre, C.C., and Lisman, J.E. (2007). Reversal of synaptic memory by $\mathrm{Ca} 2+/$ calmodulin-dependent protein kinase II inhibitor. J Neurosci 27, 5190-5199.

Scanziani, M., Malenka, R.C., and Nicoll, R.A. (1996). Role of intercellular interactions in heterosynaptic long-term depression. Nature 380, 446-450.

Schnell, E., Sizemore, M., Karimzadegan, S., Chen, L., Bredt, D.S., and Nicoll, R.A. (2002). Direct interactions between PSD-95 and stargazin control synaptic AMPA receptor number. Proc Natl Acad Sci U S A 99, 13902-13907.

Schulman, H. (1984). Phosphorylation of microtubule-associated proteins by a $\mathrm{Ca} 2+/$ calmodulin-dependent protein kinase. J Cell Biol 99, 11-19.

Silva, A.J., Stevens, C.F., Tonegawa, S., and Wang, Y. (1992). Deficient hippocampal long-term potentiation in alpha-calcium-calmodulin kinase II mutant mice. Science 257, 201-206.

Stein, I.S., Park, D.K., Claiborne, N., and Zito, K. (2021). Non-ionotropic NMDA receptor signaling gates bidirectional structural plasticity of dendritic spines. Cell Rep 34, 108664.

Stevens, C.F., Tonegawa, S., and Wang, Y. (1994). The role of calciumcalmodulin kinase II in three forms of synaptic plasticity. Curr Biol 4, 687-693.

Stoppini, L., Buchs, P.A., and Muller, D. (1991). A simple method for organotypic cultures of nervous tissue. J Neurosci Methods 37, 173-182.

Strack, S., Barban, M.A., Wadzinski, B.E., and Colbran, R.J. (1997a). Differential inactivation of postsynaptic density-associated and soluble $\mathrm{Ca} 2+$ /calmodulindependent protein kinase II by protein phosphatases 1 and 2A. J Neurochem 68, 2119-2128.

Strack, S., Choi, S., Lovinger, D.M., and Colbran, R.J. (1997b). Translocation of autophosphorylated calcium/calmodulin-dependent protein kinase II to the postsynaptic density. J Biol Chem 272, 13467-13470.

Stratton, M., Lee, I.H., Bhattacharyya, M., Christensen, S.M., Chao, L.H., Schulman, H., Groves, J.T., and Kuriyan, J. (2014). Activation-triggered subunit exchange between CaMKII holoenzymes facilitates the spread of kinase activity. Elife 3, e01610.

Toyoizumi, T., Kaneko, M., Stryker, M.P., and Miller, K.D. (2014). Modeling the dynamic interaction of Hebbian and homeostatic plasticity. Neuron 84, 497-510.

Trinidad, J.C., Thalhammer, A., Specht, C.G., Schoepfer, R., and Burlingame, A.L. (2005). Phosphorylation state of postsynaptic density proteins. J Neurochem 92, 1306-1316.

Turrigiano, G.G. (2008). The self-tuning neuron: synaptic scaling of excitatory synapses. Cell 135, 422-435.

Vest, R.S., Davies, K.D., O'Leary, H., Port, J.D., and Bayer, K.U. (2007). Dual mechanism of a natural CaMKII inhibitor. Mol Biol Cell 18, 5024-5033. 
1033 Wang, J.H., and Kelly, P.T. (1996). The balance between postsynaptic Ca(2+)1034 dependent protein kinase and phosphatase activities controlling synaptic strength. 1035 Learn Mem 3, 170-181.

1036 Wang, X.J. (2001). Synaptic reverberation underlying mnemonic persistent 1037 activity. Trends Neurosci 24, 455-463.

1038 Wong, J.M., and Gray, J.A. (2018). Long-Term Depression Is Independent of 1039 GluN2 Subunit Composition. J Neurosci 38, 4462-4470.

1040 
Fig.1

A

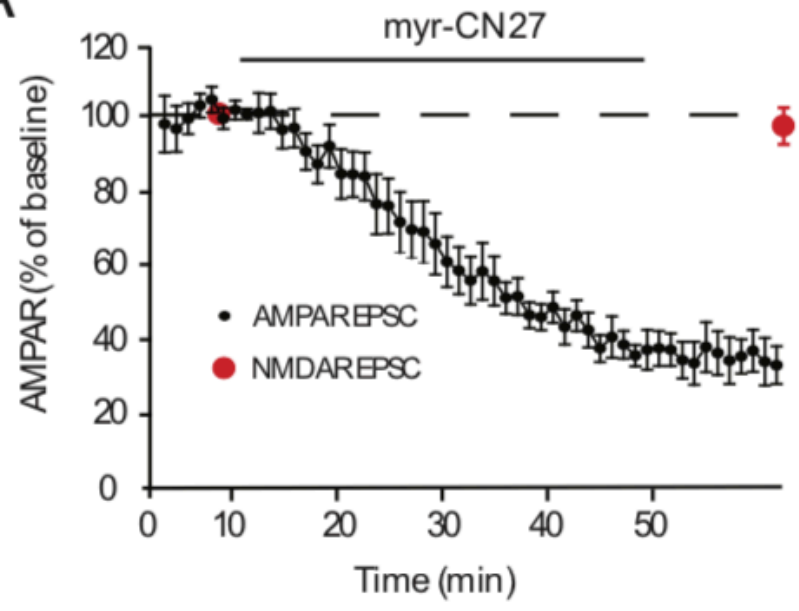

B

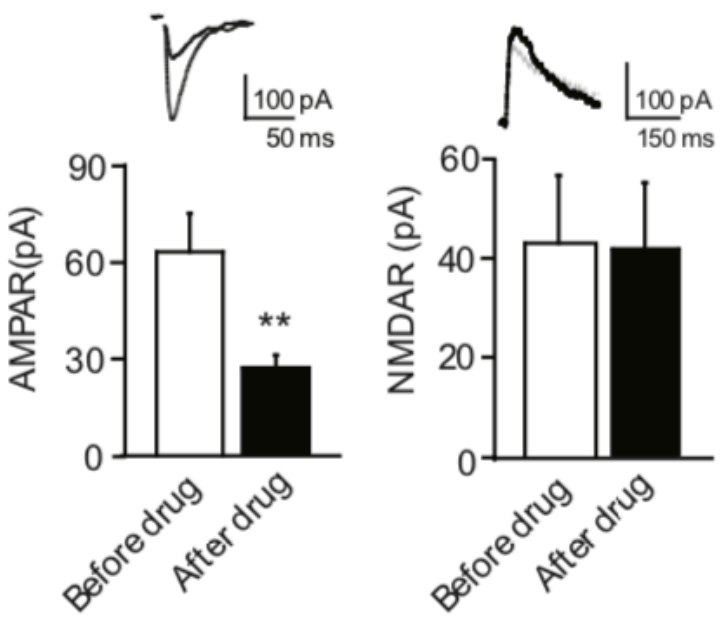

D

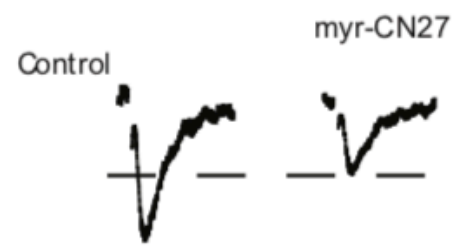

CASPRCAMKI
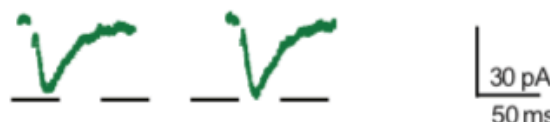

E

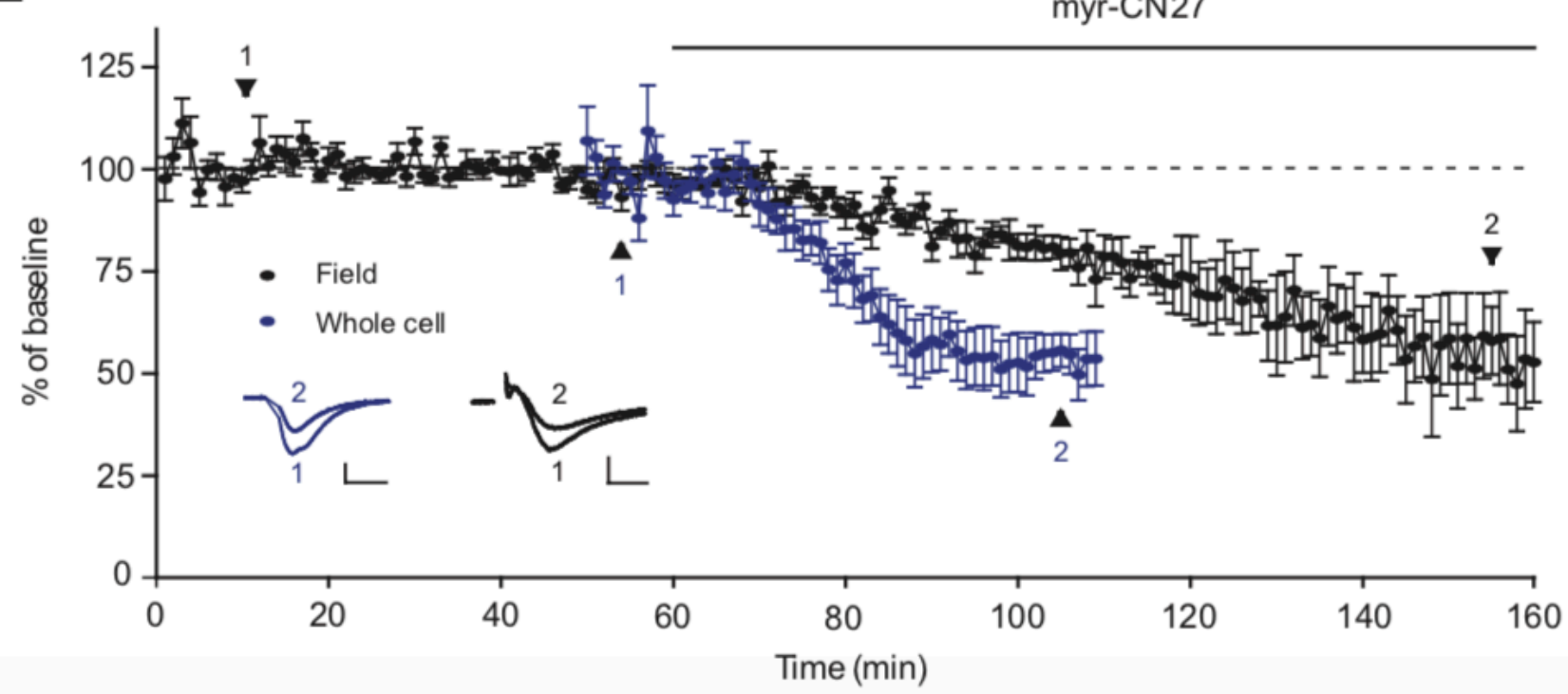







Fig.2

A



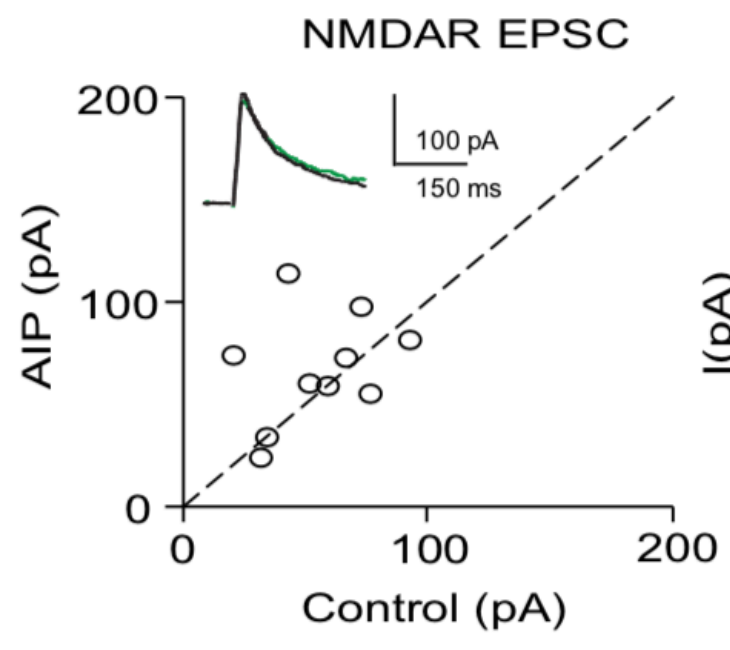

B

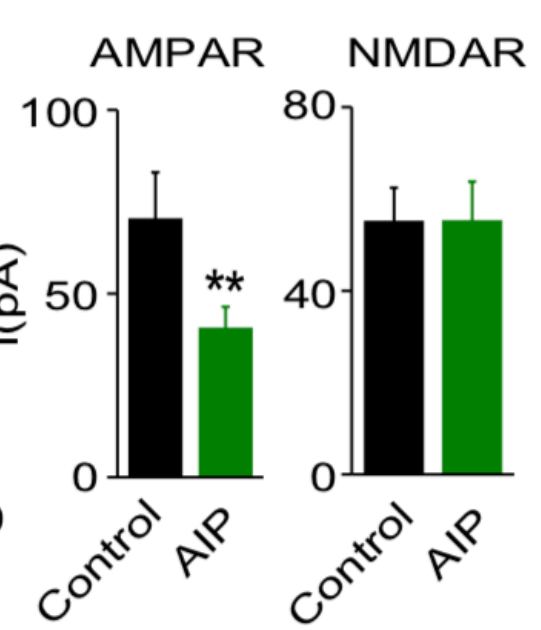

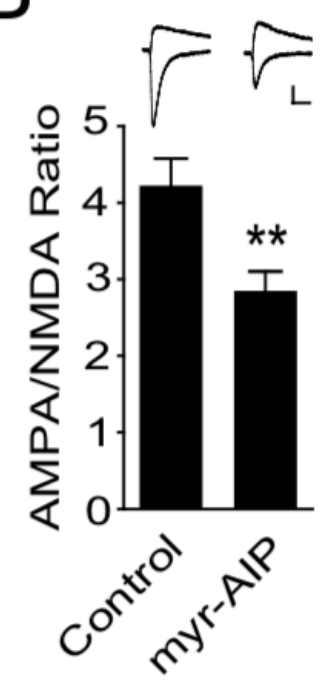

C



myr-AIP
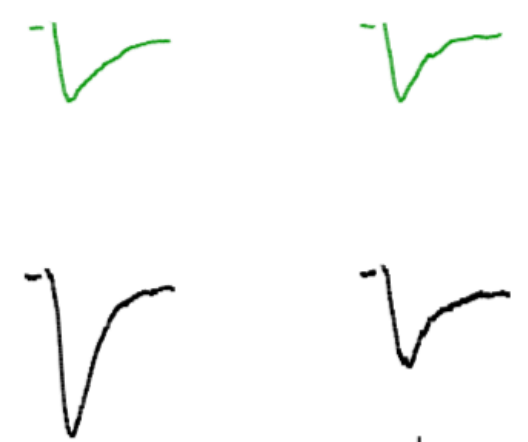

$150 \mathrm{pA}$

$50 \mathrm{~ms}$ 
Fig.3



B
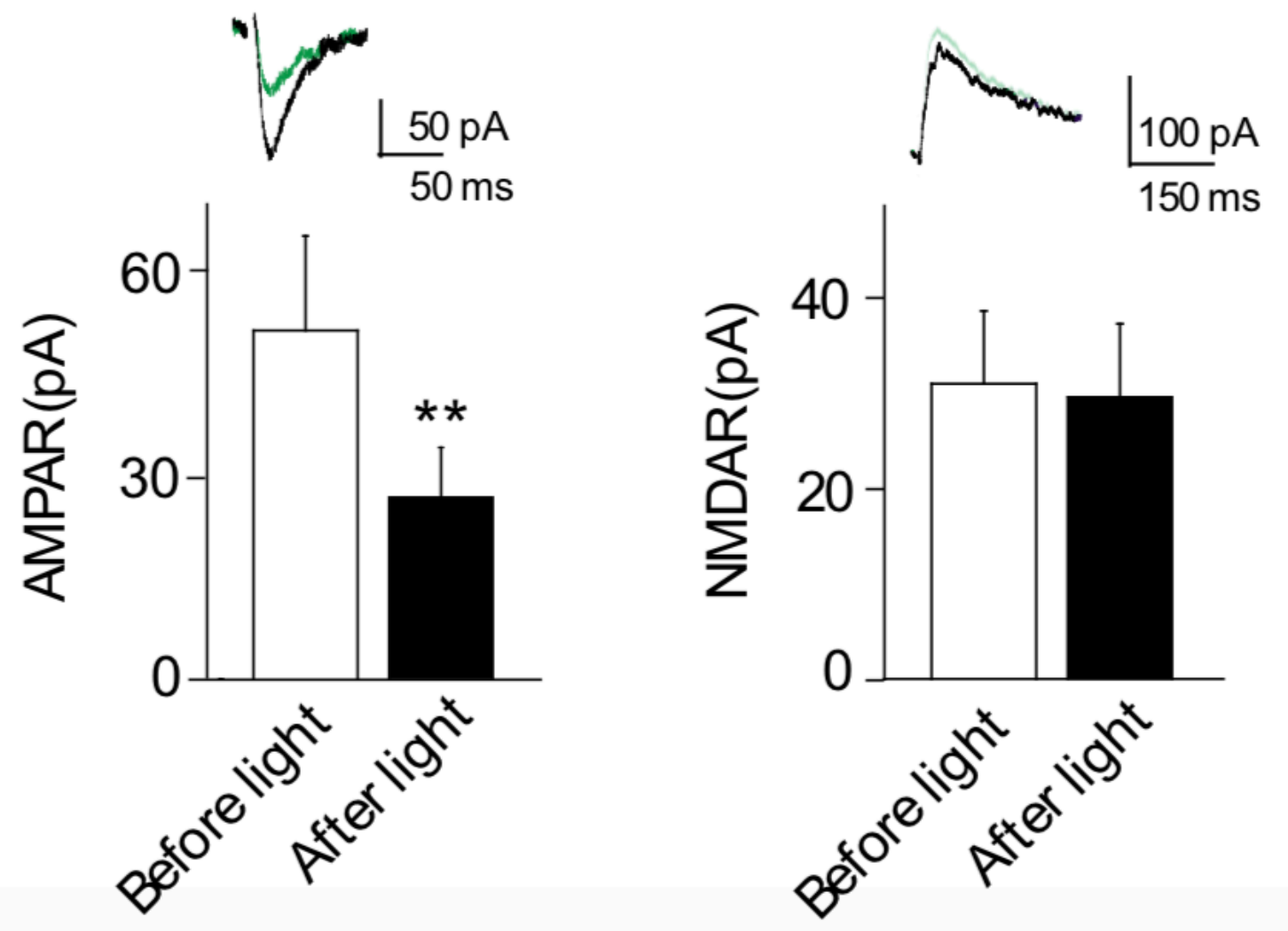
Fig. S2

\section{Before light}
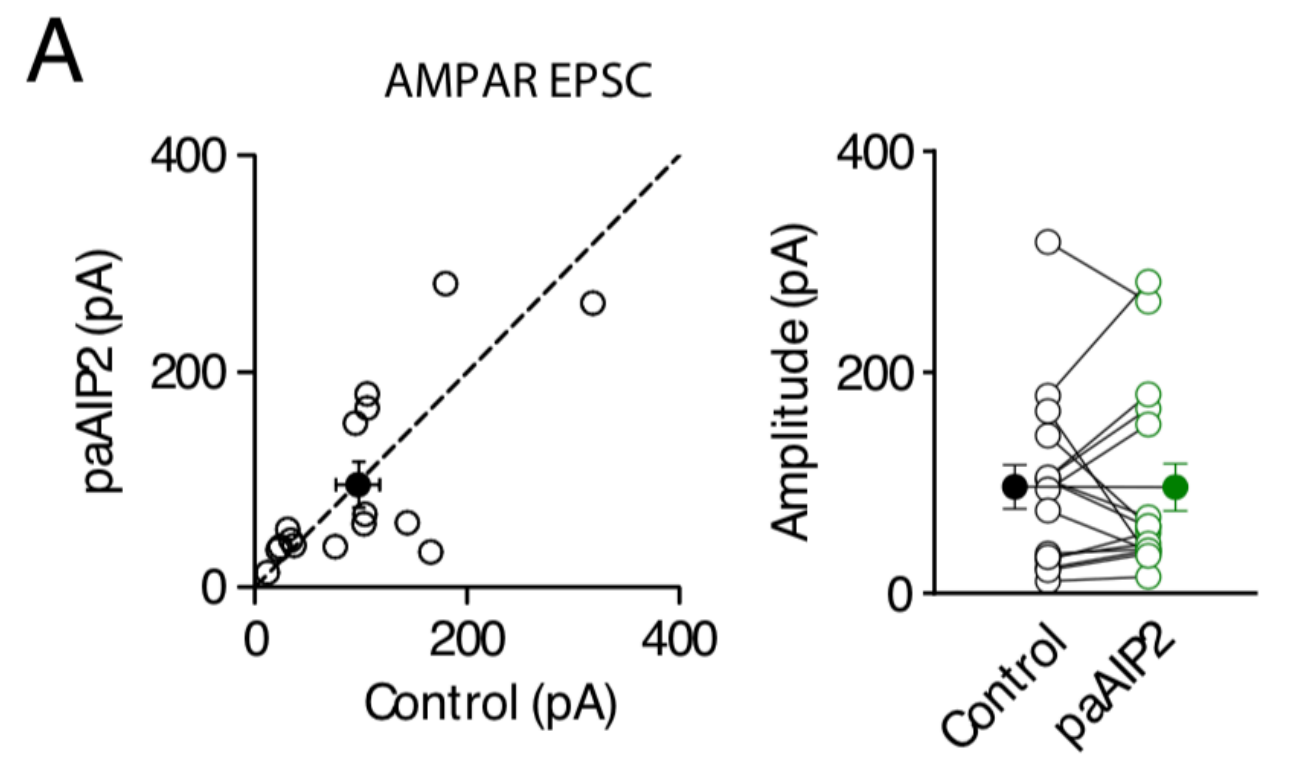

\section{After light}

C

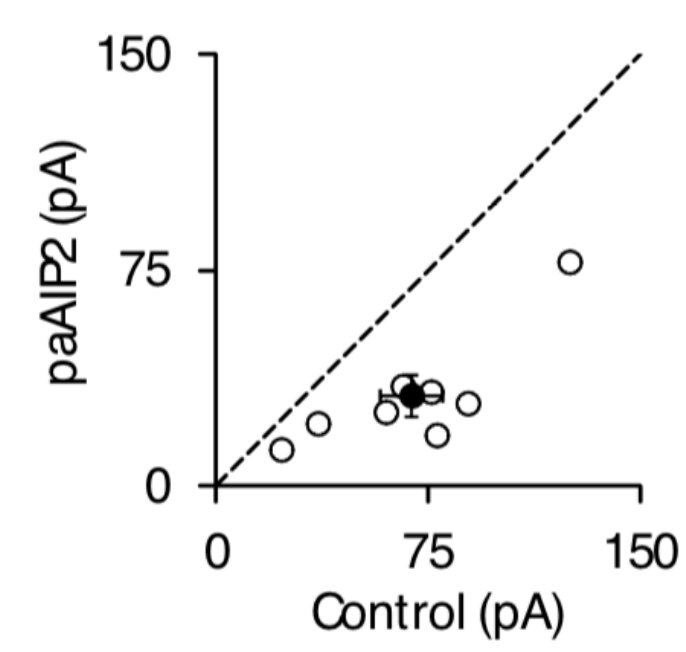

AMPAR EPSC

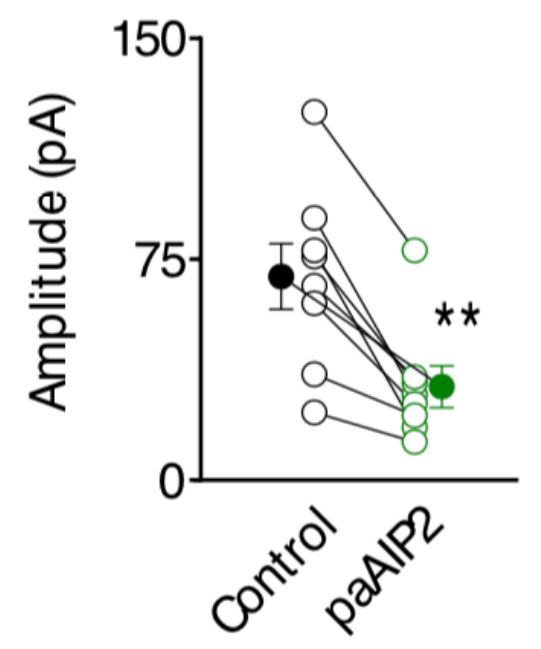

B
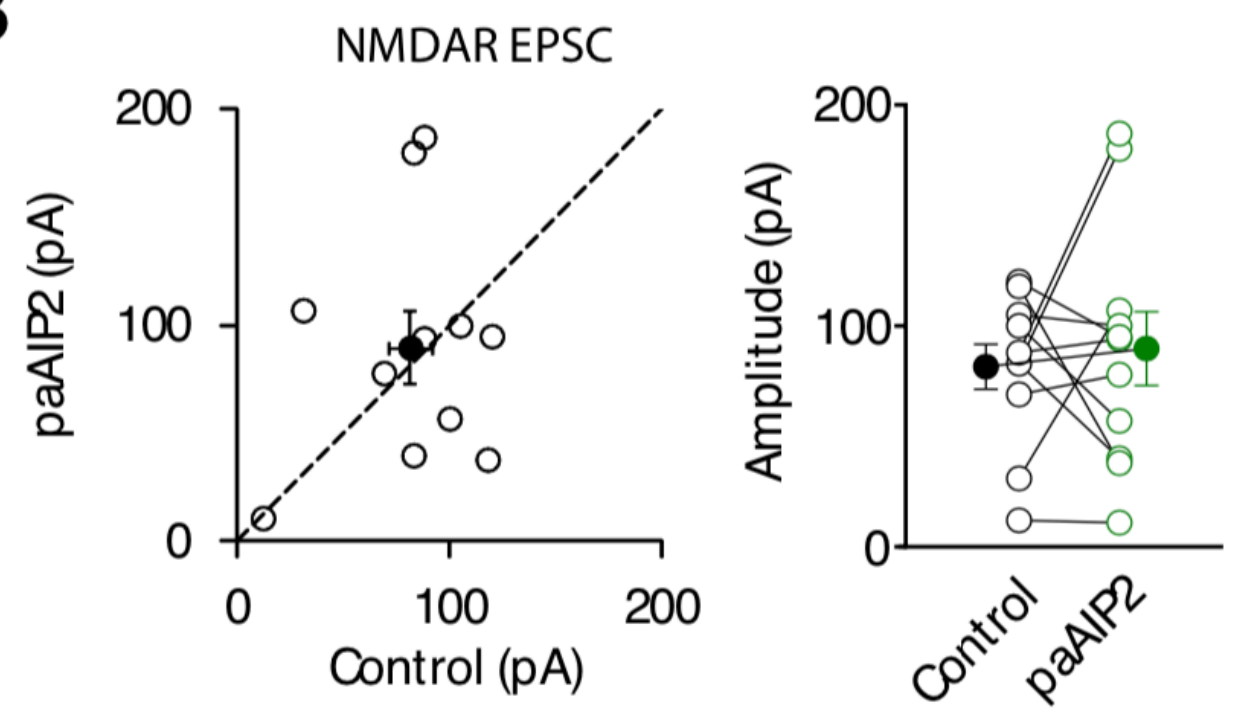

D
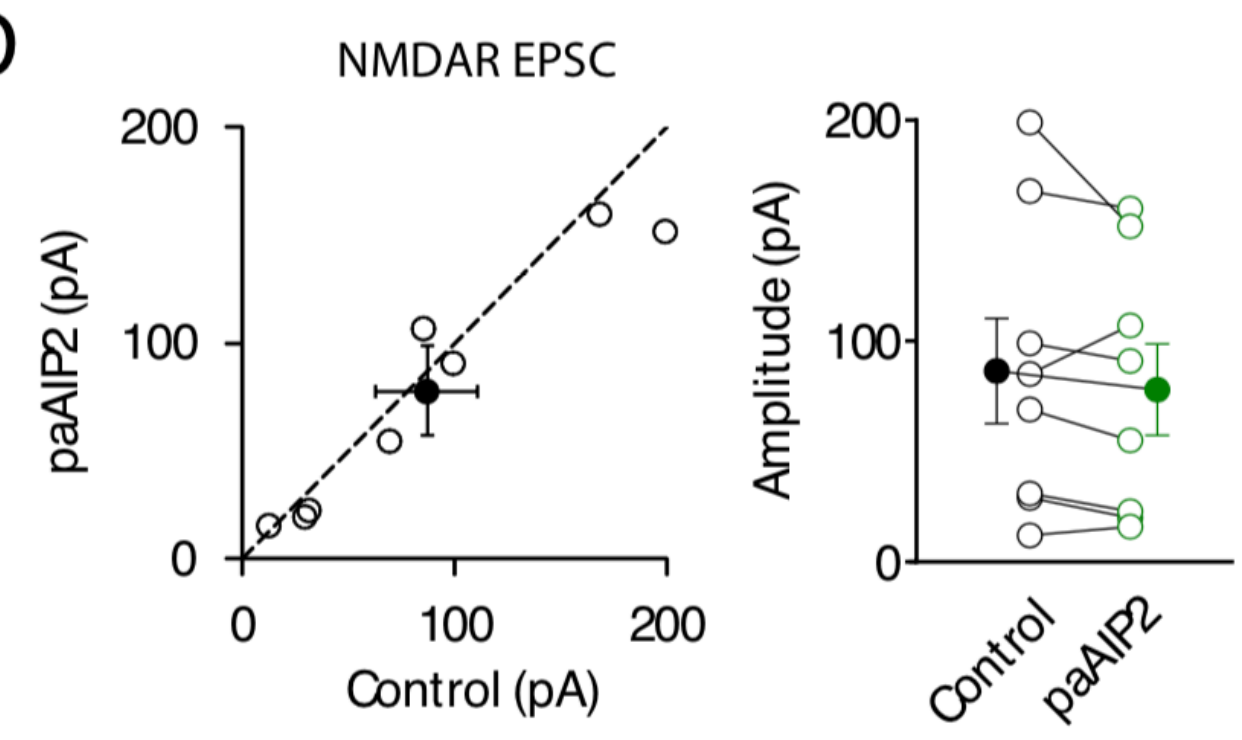
Fig. S3

\section{CRISPR- CaMKIl $\alpha$}

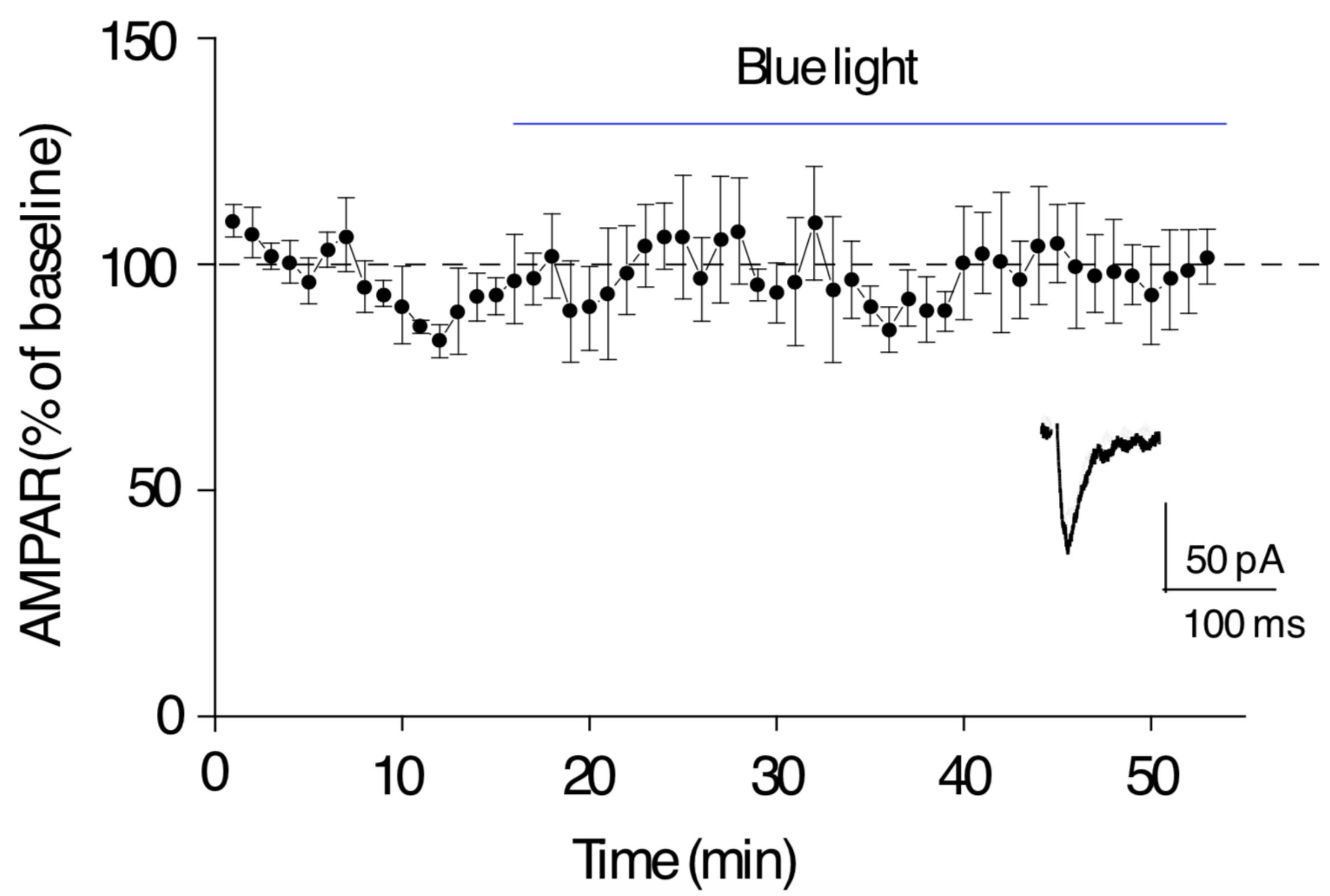


Fig.4
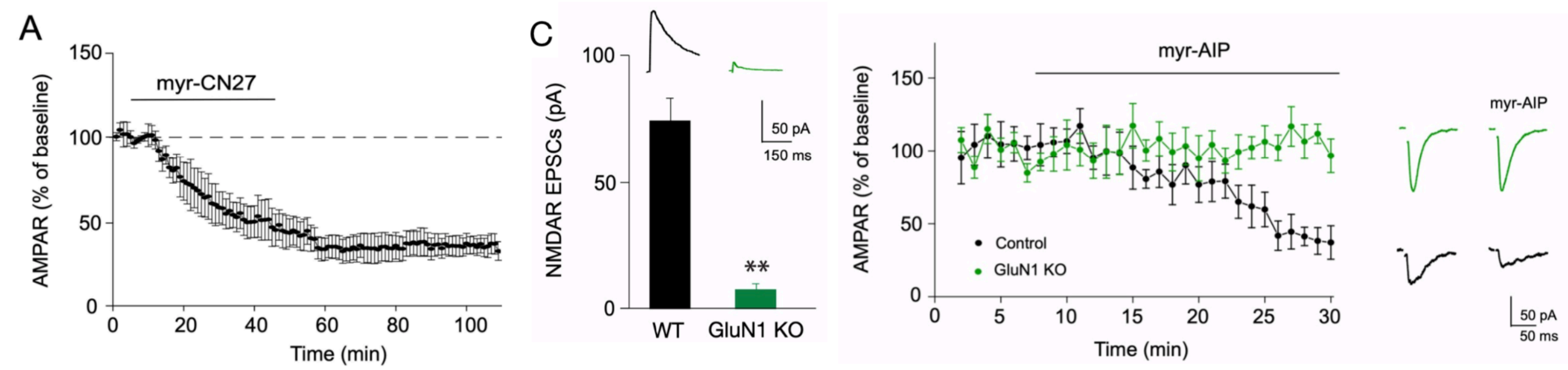

B
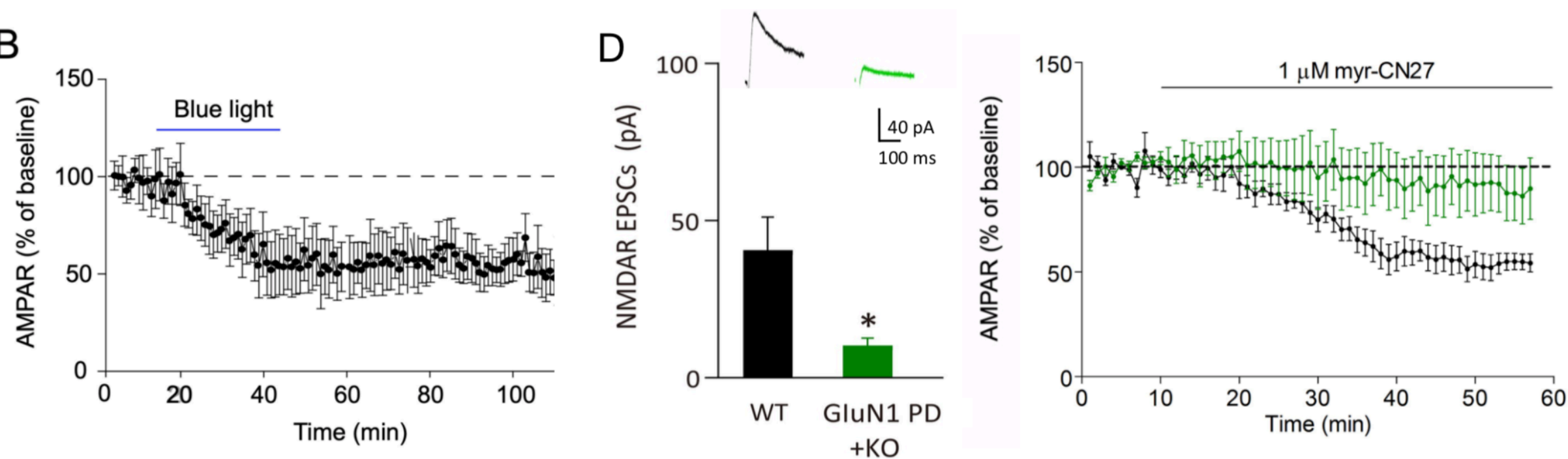

myr-CN27

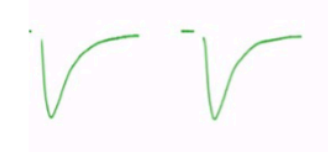


Fig. S4
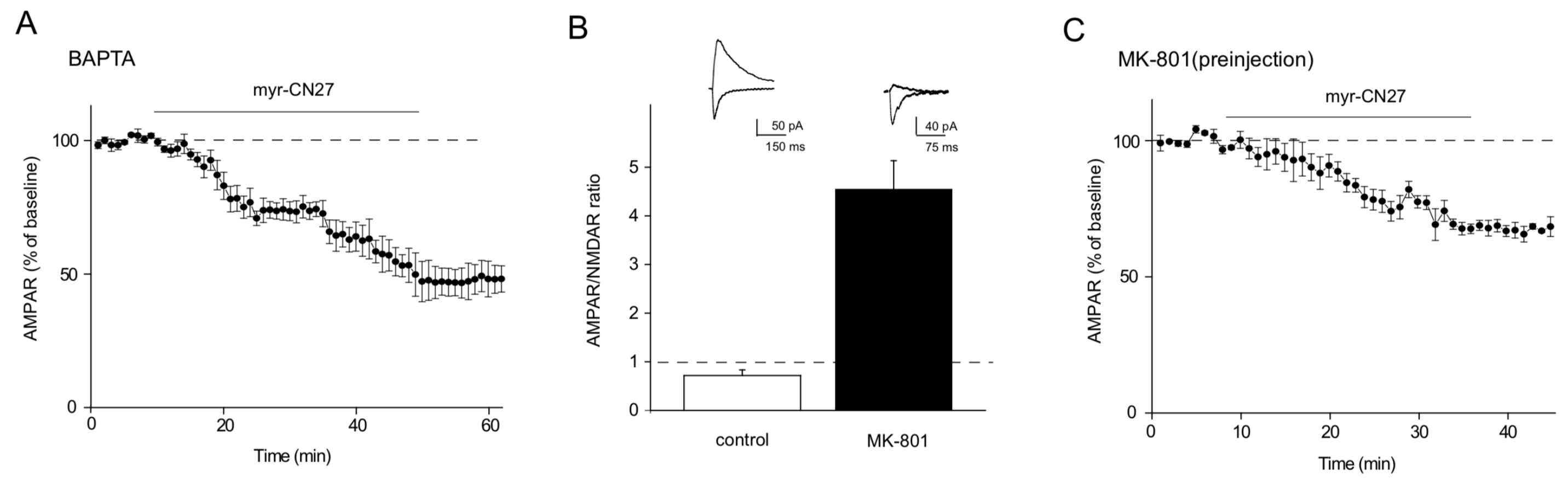
Fig. 5

A PaAIP2
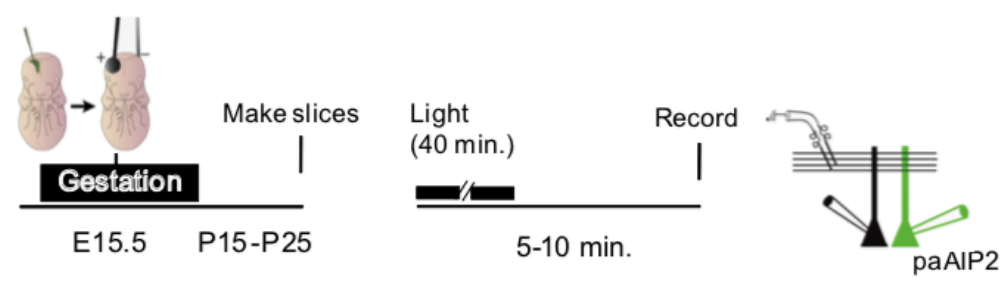

B
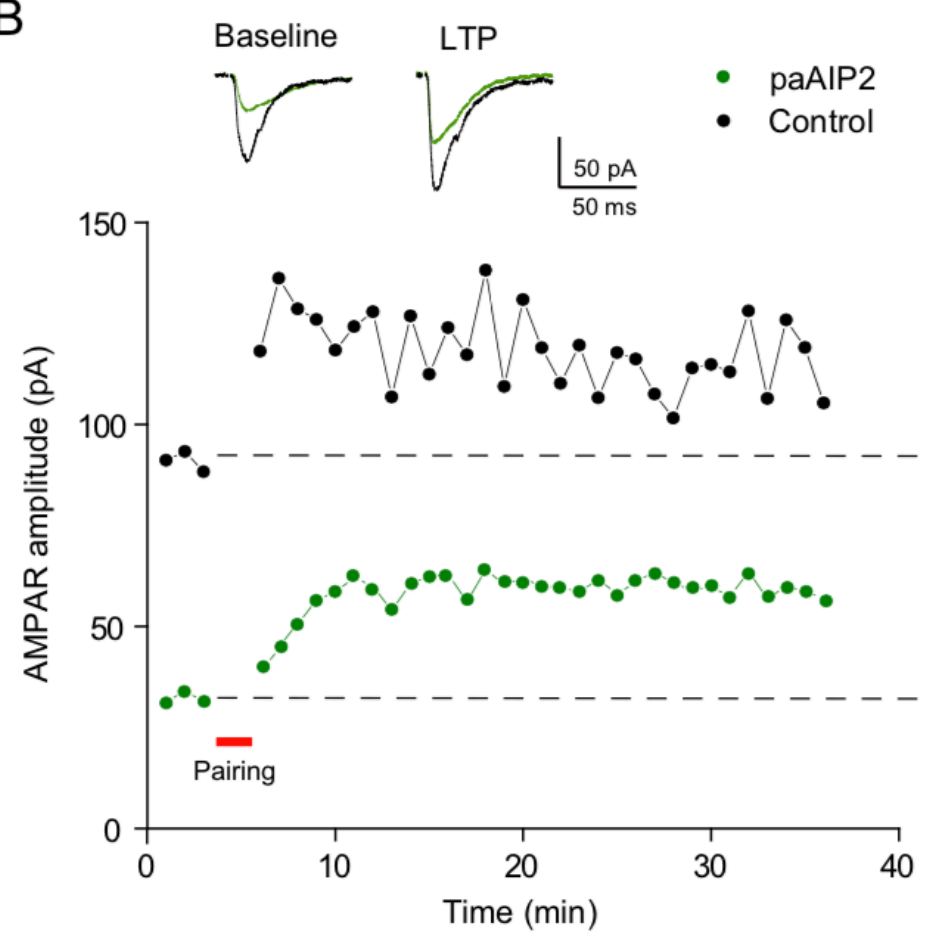

D

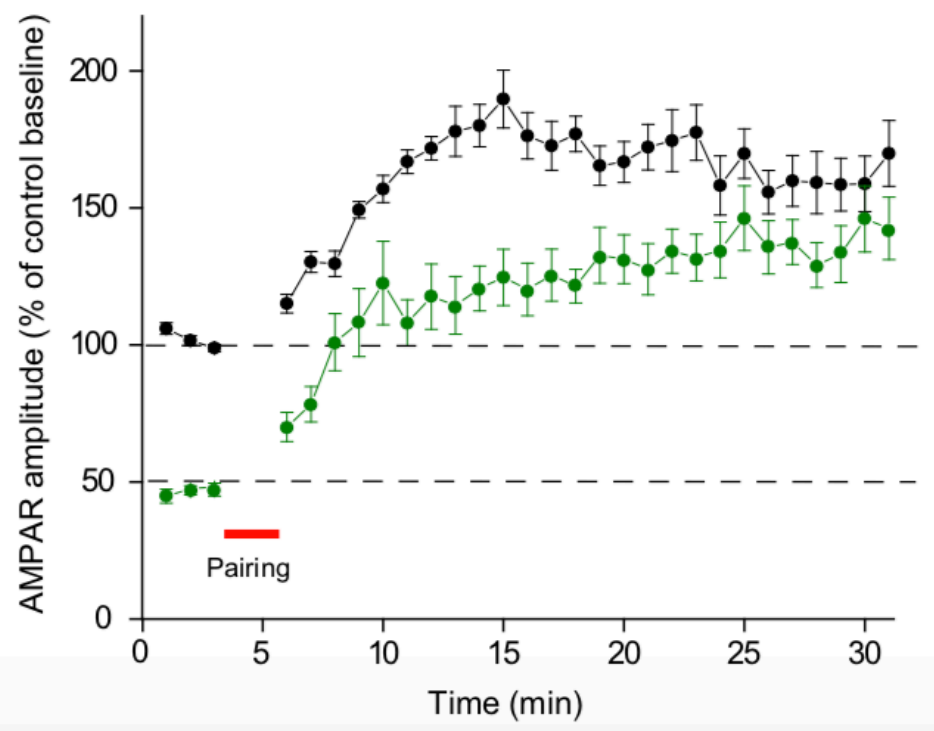

C

Normalized

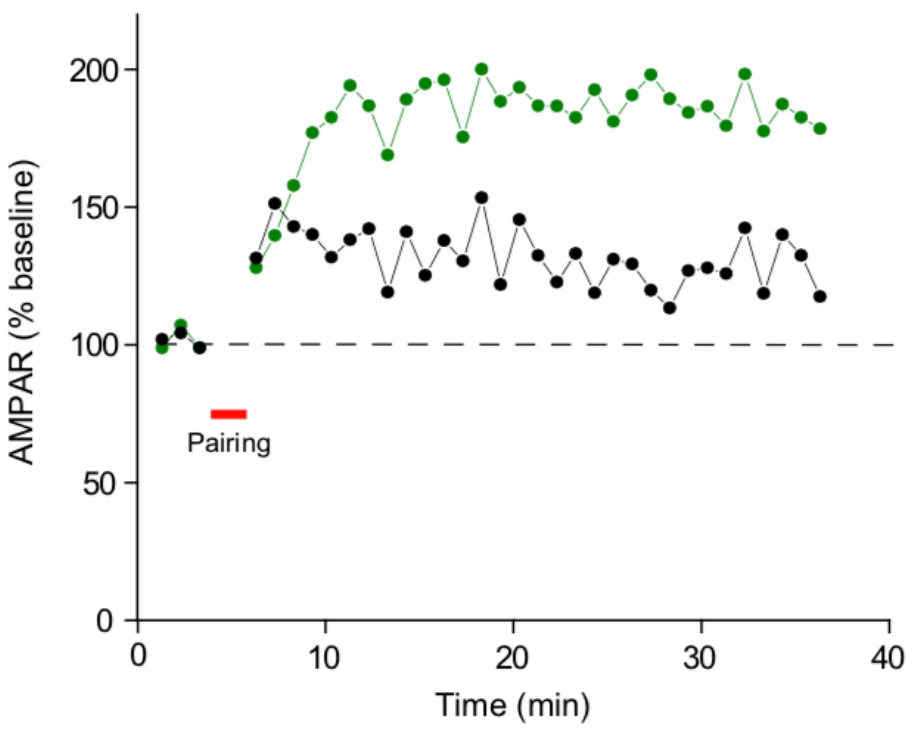

E

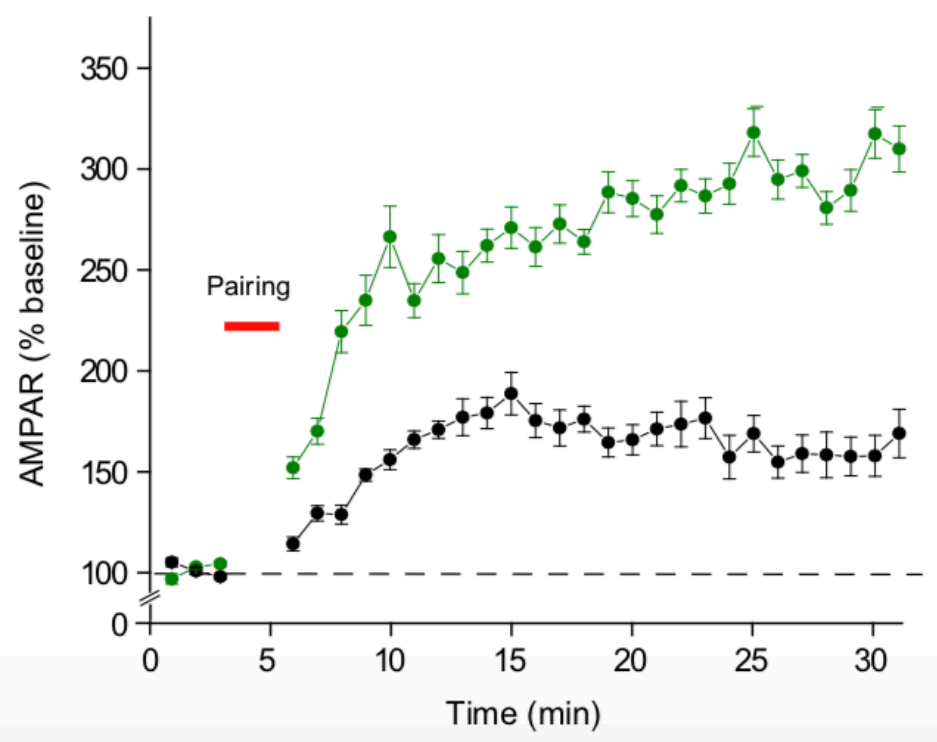


Fig. 6

A

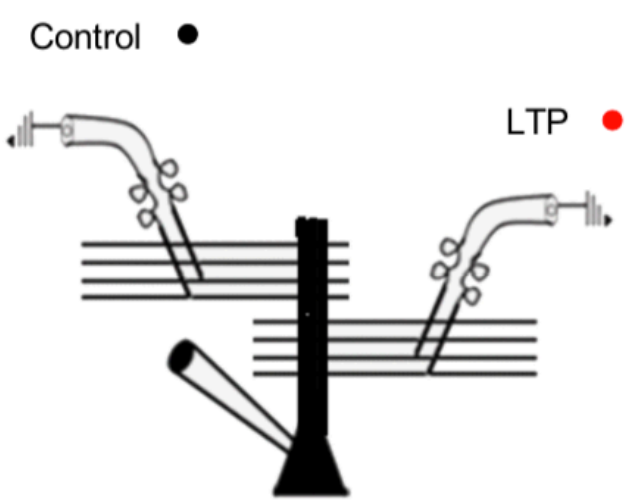

2 - pathway experiment
B

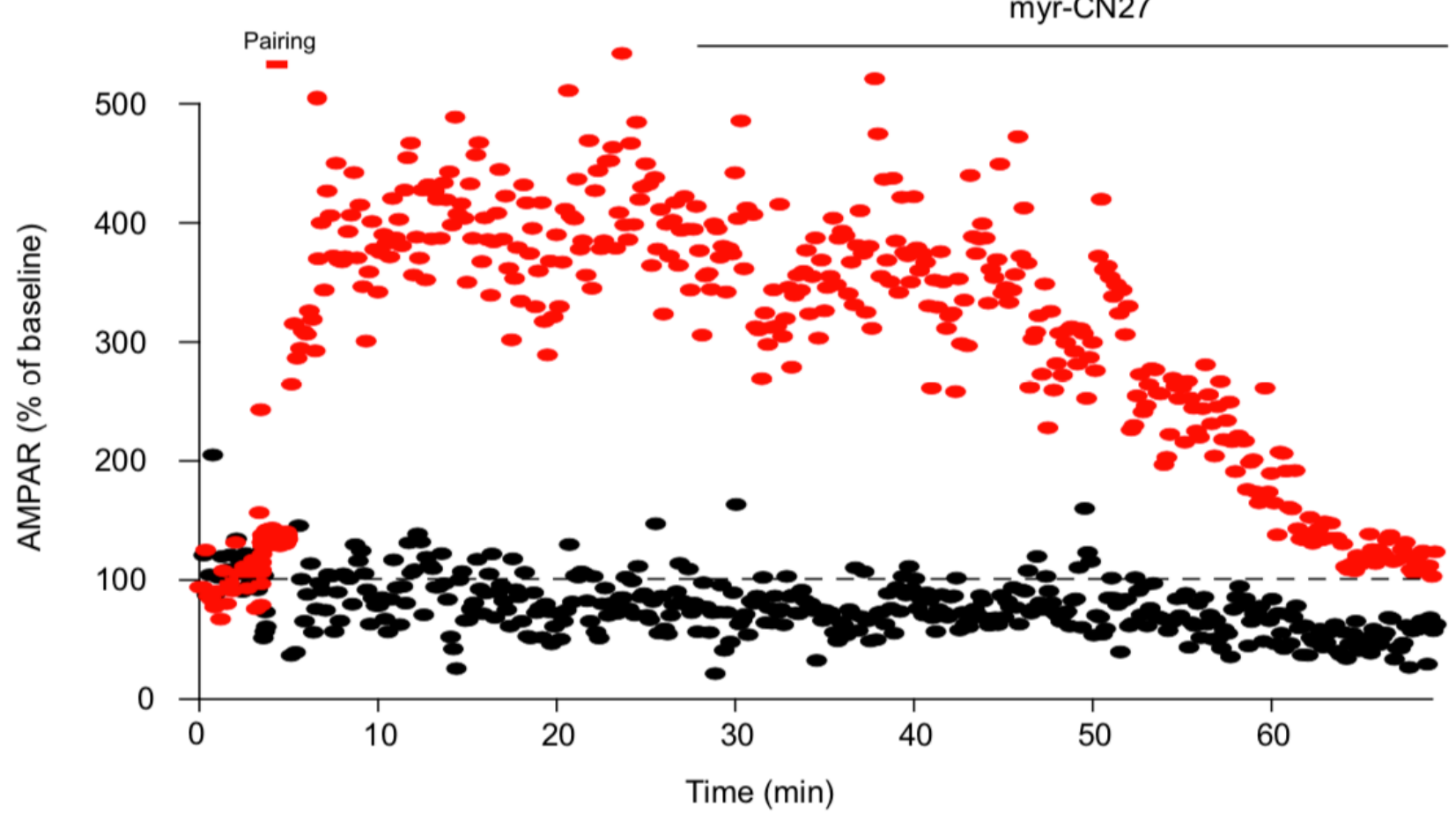

C

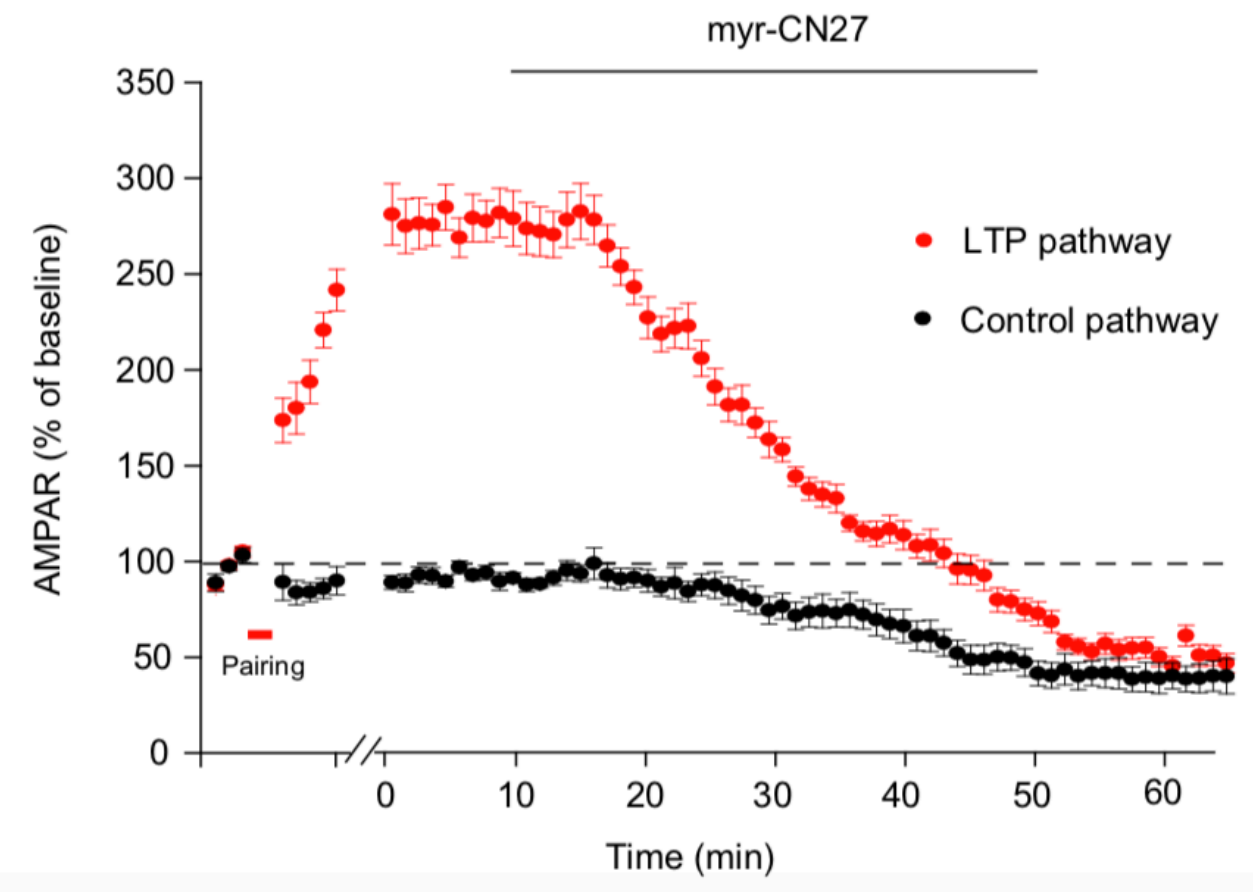

Before LTP

After LTP

myr-CN27

LTP pathway

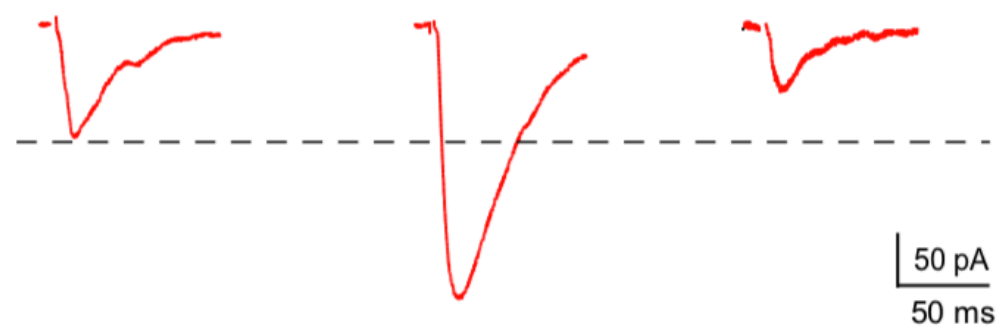

Control pathway

Fig. S5

Two Pathway LTP with myr-CN27 application

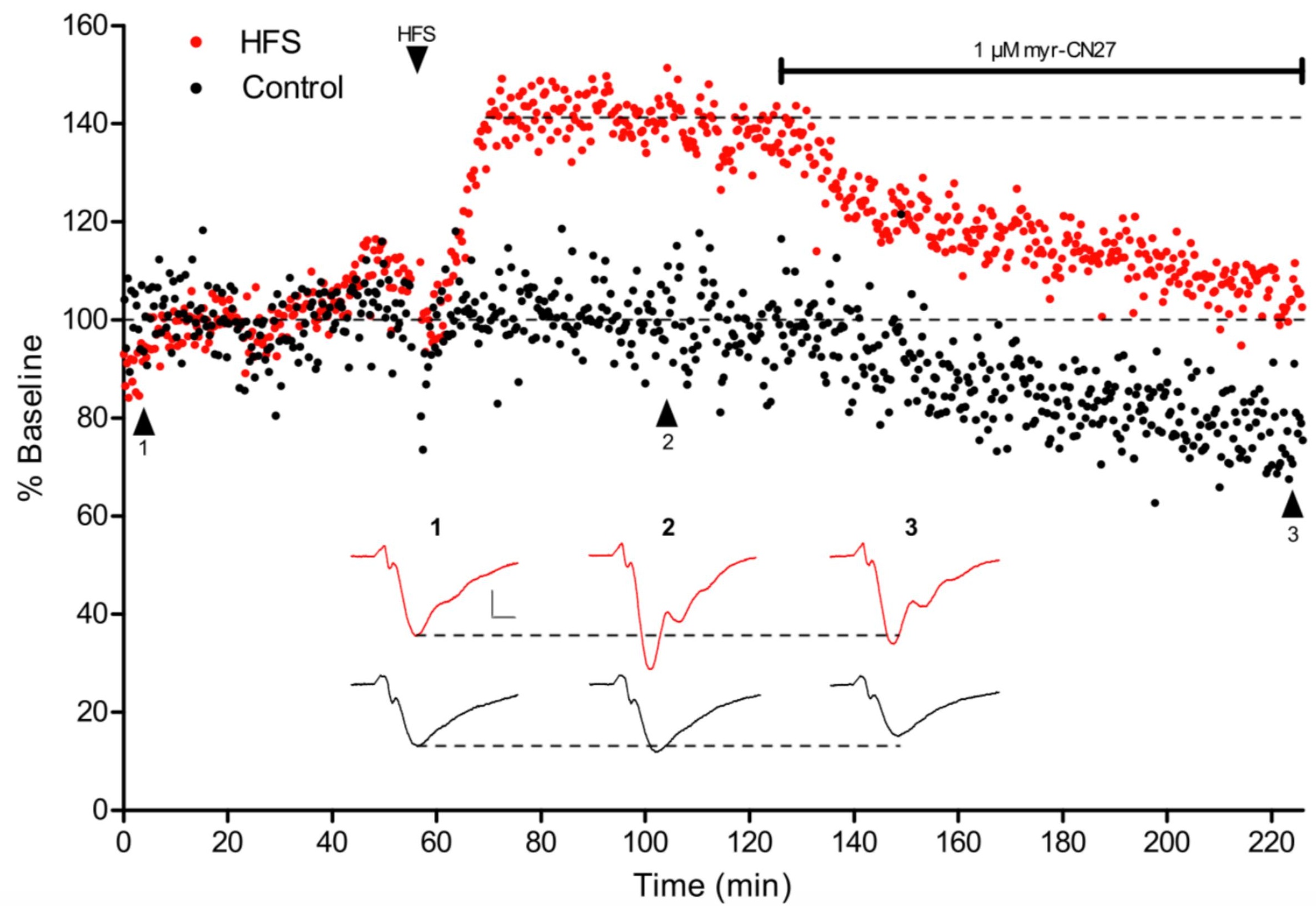

